\title{
XMM-NEWTON OBSERVATIONS OF THE NARROW-LINE SEYFERT 1 GALAXY Mrk 335 IN A HISTORICAL LOW X-RAY FLUX STATE
}

\author{
Dirk Grupe, ${ }^{1}$ Stefanie Komossa, ${ }^{2}$ Luigi C. Gallo, ${ }^{3}$ Andrew C. Fabian, ${ }^{4}$ Josefin Larsson, ${ }^{4}$ \\ Anil K. Pradhan, ${ }^{5}$ Dawei Xu, ${ }^{6}$ and Giovanni Miniutti ${ }^{4}$ \\ Received 2007 December 3; accepted 2008 March 11
}

\begin{abstract}
We report the discovery of strong soft X-ray emission lines and a hard continuum above $2 \mathrm{keV}$ in the narrow-line Seyfert 1 galaxy Mrk 335 during an extremely low X-ray flux state. Mrk 335 was observed for 22 ks by XMM-Newton in 2007 July as a Target of Opportunity to examine it in its X-ray low flux state, which was discovered with Swift. Long-term light curves suggest that this is the lowest flux state this AGN has ever been seen in. However, Mrk 335 is still sufficiently bright that its X-ray properties can be studied in detail. The X-ray continuum spectrum is very complex and requires several components to model. Statistically, partial covering and blurred reflection models work well. We confirm the presence of a strong narrow Fe line at $6.4 \mathrm{keV}$. High-resolution spectroscopy with the $X M M$ Newton RGS reveals strong, soft X-ray emission lines not detected in previous, higher signal-to-noise ratio, $X M M$ Newton observations, such as highly ionized Fe lines, $\mathrm{O}$ VII, and $\mathrm{Ne}$ IX and $\mathrm{Mg}$ XI lines. The optical/UV fluxes are similar to those previously measured with Swift. Optical spectroscopy taken in 2007 September does not show any changes to optical spectra obtained 8 years earlier.
\end{abstract}

Subject headings: galaxies: active — galaxies: individual (Mrk 335) — galaxies: Seyfert — ultraviolet: galaxies — X-rays: galaxies

Online material: color figures

\section{INTRODUCTION}

The narrow-line Seyfert 1 galaxy (NLS1; Osterbrock \& Pogge 1985) Mrk $335\left(\alpha_{\mathrm{J} 2000.0}=00^{\mathrm{h}} 06^{\mathrm{m}} 19.5^{\mathrm{s}}, \delta_{\mathrm{J} 2000.0}=\right.$ $\left.+20^{\circ} 12^{\prime} 11.0^{\prime \prime}, z=0.026\right)$ was discovered as a bright X-ray active galactic nucleus (AGN) with Uhuru (Tananbaum et al. 1978) in 1971. Consequently, it has been a target of almost all X-ray observatories, including Einstein, EXOSAT, Ginga, Röntgensatellit (ROSAT), ASCA, XMM-Newton, and Suzaku (Halpern 1982; Pounds et al. 1987; Nandra \& Pounds 1994; Grupe et al. 2001; George et al. 2000; Gondoin et al. 2002; Longinotti et al. 2007b, 2007c; O'Neill et al. 2007; Larsson et al. 2008). The X-ray spectrum of Mrk 335 appears to be rather complicated and cannot be modeled by a single power-law spectrum. Possible interpretations for the X-ray spectrum include absorption by ionized gas (e.g., Nandra \& Pounds 1994; Leighly 1999b), partial covering (e.g., Tanaka et al. 2005), and X-ray reflection on the accretion disk (e.g., Ballantyne et al. 2001; Crummy et al. 2006; Longinotti et al. 2007b; O'Neill et al. 2007; Larsson et al. 2008). Support for the reflection interpretation comes from the presence of a broadened and asymmetric $\mathrm{Fe} \mathrm{K} \alpha$ line (Longinotti et al. 2007b; O'Neill et al. 2007). Such lines are predicted as a consequence of reflection of the primary power law on the accretion disk close to the black hole (Fabian et al. 1989) and are seen in a growing number of AGNs (see Nandra et al. 2007 and

\footnotetext{
1 Department of Astronomy and Astrophysics, Pennsylvania State University, University Park, PA 16802; grupe@astro.psu.edu.

2 Max-Planck-Institut für extraterrestrische Physik, D-85748 Garching, Germany; skomossa@mpe.mpg.de.

3 Department of Astronomy and Physics, Saint Mary's University, Halifax, NS B3H 3C3, Canada; lgallo@ap.stmarys.ca.

4 Institute of Astronomy, Cambridge, CB3 0HA, UK.

5 Department of Astronomy, Ohio State University, Columbus, OH 43210; pradhan@astronomy.ohio-state.edu.

${ }^{6}$ National Astronomical Observatories, Chinese Academy of Science, Beijing 100012, China; dwxu@bao.ac.cn.
}

references therein). Mrk 335 is a highly variable X-ray source that typically varies by factors of $2-4$ on hourly timescales (e.g., Turner et al. 1993; O’Neill et al. 2007).

However, when Mrk 335 was observed with the Gamma-Ray Burst Explorer Mission Swift (Gehrels et al. 2004) in 2007 May and June (Grupe et al. 2007a), it appeared significantly fainter in its X-ray flux by factors of more than 30 compared to the 2006 XMM-Newton and Suzaku observations (Longinotti et al. 2007c; O'Neill et al. 2007; Larsson et al. 2008). This drop in X-rays coincided with substantial changes in the X-ray spectrum. Mrk 335 showed a very hard X-ray component at energies above $2 \mathrm{keV}$, very similar to what has been seen in other NLS1 galaxies (e.g., Gallo 2006) and specifically in NGC 4051 (Guainazzi et al. 1998; Ponti et al. 2006).

As discussed in Grupe et al. (2007a), one possible explanation for the sudden drop in the X-ray flux of Mrk 335 is a partial covering absorber that intersects our line of sight with the X-rayemitting zone. Grupe et al. (2007a) found that when correcting for this absorber the intrinsic X-ray flux of Mrk 335 between the high and low flux states varied only by factors of 4-6.

In order to investigate the nature of the low state in more detail, we initiated a Target of Opportunity ( $\mathrm{ToO}$ ) observation with XMM-Newton, which was executed on 2007 July 10. Prior to this, Mrk 335 was observed by XMM-Newton in 2000 and 2006 (Gondoin et al. 2002; Crummy et al. 2006; Longinotti et al. 2007b; O'Neill et al. 2007). Of interest with Mrk 335 is the broad component in the spectrum associated with the red wing of a relativistic iron line. This wing is not required if the spectrum is modified by a partial covering absorber. The brightness of Mrk 335 provides us with the rare opportunity to study an AGN in its low state, but still with sufficient signal to obtain wellexposed spectra in a relatively short observation. In this paper we report the XMM-Newton ToO observation.

The paper is organized as follows: in $\S 2$ we present the $X M M$ Newton and Swift observations and describe the data reduction, 
and in $\S 3$ we present the results that are discussed in $\S 4$. Throughout the paper spectral indices are denoted as energy spectral indices with $F_{\nu} \propto \nu^{-\alpha}$. Luminosities are calculated assuming a $\Lambda \mathrm{CDM}$ cosmology with $\Omega_{M}=0.27, \Omega_{\Lambda}=0.73$, and a Hubble constant of $H_{0}=75 \mathrm{~km} \mathrm{~s}^{-1} \mathrm{Mpc}^{-1}$ corresponding to a luminosity distance $D=105 \mathrm{Mpc}$. All errors are $90 \%$ confidence unless stated otherwise.

\section{OBSERVATIONS AND DATA REDUCTION}

\subsection{XMM-Newton and Swift Observations}

XMM-Newton (Jansen et al. 2001) observed Mrk 335 on 2007 July 10 for a total of $22 \mathrm{ks}$ (ObsID 0510010701). A summary of the observations with each of the instruments on board XMMNewton is given in Table 1. The European Photon Imaging Camera (EPIC) pn (Strüder et al. 2001) was operated in large window mode with the thin filter. This combination was chosen to avoid pileup in case the AGN rebrightened. The two EPIC MOS cameras (Turner et al. 2001) were both operated in full frame mode with the medium filters. High-resolution X-ray spectroscopy was performed using the two Reflection Grating Spectrometers (RGS; den Herder et al. 2001) on board XMM-Newton. Optical photometry was performed in five filters with the Optical Monitor (OM; Mason et al. 2001). The data are used to measure the opticalto-X-ray spectral energy distribution (SED) of Mrk 335 during the $X M M$-Newton observation. Due to slew problems at the beginning of the observations, $V$ filter observations were not obtained. All OM observations were performed in a science-user-defined configuration with a $7^{\prime} \times 7^{\prime}$ observing window.

The XMM-Newton data were processed in the standard way using the XMMSAS version xmmsas_20060628_1801-7.0.0. The EPIC pn data were checked for episodes of high particle background. The background count rate was found low throughout the observation. The source X-ray photons in the EPIC pn were selected in a circular region with a radius of $1^{\prime}$. Likewise, background photons were selected from a nearby, source-free region with the same radius. Only single and double events (PATTERN.le.4) and single to quadruple events (PATTERN.le.12) were selected for the pn and MOS data, respectively. The spectra were rebinned with grppha version 3.0 .0 with 50 photons bin $^{-1}$. The redistribution matrices and the auxiliary response files were created by the XMMSAS tasks rmfgen and arfgen, respectively. RGS spectra and response matrixes were created by the standard RGS XMMSAS tool rgsproc. The RGS spectra were rebinned with 10 photons bin $^{-1}$ using grppha. Spectral fits to the EPIC pn and MOS and RGS spectra were performed with XSPEC version 12.3.1x (Arnaud 1996). The OM data were processed with the XMMSAS task omichain. The magnitudes and fluxes of Mrk 335 were taken from the source lists created by the omichain task. For the count rate-to-flux conversion we used the conversion factors given in the OM calibration document XMM-SOC-CAL-TN-0019.

The Swift X-Ray Telescope (XRT; Burrows et al. 2005) and UV/Optical Telescope (UVOT; Roming et al. 2005) 2007 May and June observations and the data reductions are described in Grupe et al. (2007a). In order to compare the photometry in the OM with the Swift UVOT, we selected five field stars with similar brightness in $V$ as Mrk 335. The measured (uncorrected) magnitudes of these stars are listed in Table 2. We confirm the earlier result by Grupe et al. (2007a) that in $U$ the magnitudes in the OM and UVOT agree perfectly. However, the OM magnitude had to be adjusted by $-0.10 \mathrm{mag}$ in $B$ and $+0.30 \mathrm{mag}$ in the UVM2 filter. In addition to the $2000 X M M$-Newton observation, during the 2007 XMM-Newton ToO observation we also observed
TABLE 1

Summary of the 2007 July 10 XMM-Newton Observations of Mrk 335

\begin{tabular}{|c|c|c|c|}
\hline Instrument & $\begin{array}{c}T_{\text {start }} \\
\text { (UT) }\end{array}$ & $\begin{array}{c}T_{\text {stop }} \\
\text { (UT) }\end{array}$ & $\begin{array}{l}T_{\exp } \\
\text { (s) }\end{array}$ \\
\hline EPIC pn..................... & $17: 09$ & $21: 57$ & 16779 \\
\hline EPIC MOS1 & $16: 14$ & $21: 56$ & 20348 \\
\hline EPIC MOS2 & $16: 14$ & $21: 56$ & 20351 \\
\hline RGS1 & $15: 50$ & $21: 58$ & 21932 \\
\hline RGS2 & $15: 50$ & $21: 58$ & 21936 \\
\hline OM U & $15: 36$ & $16: 32$ & 3000 \\
\hline OM B & $16: 32$ & $17: 10$ & 2000 \\
\hline 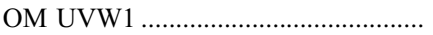 & $17: 10$ & $18: 22$ & 4000 \\
\hline 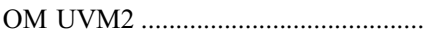 & $18: 22$ & 19:34 & 4000 \\
\hline 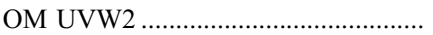 & $19: 34$ & $20: 46$ & 4000 \\
\hline OM UVW2 & $20: 46$ & $21: 57$ & 4000 \\
\hline
\end{tabular}

in UVW1 and UVW2 as listed in Table 1. In these filters the OM magnitudes have to be adjusted, compared to the Swift UVOT, by +0.78 and +0.15 mag for the UVW1 and UVW2 filters, respectively. The five stars also show that the OM and UVOT detectors are stable, and no variability is seen in these five stars. This suggests that any variability detected in the optical/UV in Mrk 335 is real. A summary of the UVOT calibration has recently been published by Poole et al. (2008).

\subsection{Optical Spectroscopic Observation}

Optical spectra of Mrk 335 were obtained with the $2.16 \mathrm{~m}$ telescope at the Xinglong Observatory, National Astronomical Observatories of China (NAOC) on 2007 September 7. Two spectra of Mrk 335 were taken with 30 and 60 minute exposures during clear weather conditions and $2^{\prime \prime}$ seeing. The spectrograph was equipped with a 600 line $\mathrm{mm}^{-1}$ grating and the $2^{\prime \prime}$ slit resulting in a resolution of $5 \AA$ in first order, covering the wavelength range between 4377 and $7120 \AA$. The optical data were reduced and analyzed in a standard way with the ESO Munich Image Data Analysis System (MIDAS, ver. 06FEBpl1.0). All line measurements were performed on the co-added spectrum after subtracting the Boroson \& Green (1992) I Zw $1 \mathrm{Fe}$ II template as described in Grupe et al. (2004c). For the $\mathrm{H} \beta$ line we subtracted the narrow component by constructing a narrow-line template from the [O III] $\lambda 5007$ line as described in Grupe et al. (2004c).

\section{RESULTS}

\subsection{Temporal Behavior}

\subsubsection{Long-Term Light Curves}

Figure 1 displays the observed $2.0-10.0 \mathrm{keV}, 0.2-2.0 \mathrm{keV}$, and UV $(2256 \AA)$ long-term light curves of Mrk 335 covering the time between 1971 and 2007 July. The X-ray fluxes and references are listed in Table 3. The UV fluxes were derived from the UV IUE and Hubble Space Telescope (HST) database by Dunn et al. (2006). To remain consistent with Dunn et al. (2006), the $X M M$-Newton OM and Swift UVOT light curves were created in the UVM2 filters, since its central wavelength is closest to $2256 \AA$. As noticed by Pounds et al. (1987), the EXOSAT data of Mrk 335 suggest that it was in an unusual X-ray low state in 1983. This seems coincident with a minimum in the UV light curve as well. However, about a year later in 1984 December, Mrk 335 was back in a typical high state. The plots in Figure 1 demonstrate that from 2007 May to July Mrk 335 was seen in a historic X-ray low state. This drop happened in less than $1 \mathrm{yr}$ since Mrk 335 was last observed in 2006 June with Suzaku (Larsson et al. 2008). In order 
TABLE 2

List of Reference Stars Used to Determine the Error in the Photometry of Mrk 335 and the Offset between the Swift UVOT and XMM-Newton OM Filters

\begin{tabular}{|c|c|c|c|c|c|c|c|c|}
\hline Object & $\alpha_{\mathrm{J} 2000.0}$ & $\delta_{\mathrm{J} 2000.0}$ & Filter & $X M M 2000$ & $X M M 2007$ & Swift $001^{\mathrm{a}}$ & Swift $002^{\mathrm{a}}$ & Swift $003-005^{\mathrm{a}}$ \\
\hline & 000622.8 & +201458.0 & $B$ & $14.94 \pm 0.01$ & $14.90 \pm 0.01$ & $14.89 \pm 0.01$ & $14.87 \pm 0.01$ & $14.86 \pm 0.01$ \\
\hline & 000622.8 & +201458.0 & UVW1 & $\ldots$ & $15.44 \pm 0.01$ & $16.27 \pm 0.02$ & $16.32 \pm 0.02$ & $16.33 \pm 0.02$ \\
\hline & 000622.8 & +201458.0 & UVM2 & $17.43 \pm 0.16$ & $17.45 \pm 0.09$ & $17.90 \pm 0.06$ & $17.91 \pm 0.05$ & $17.94 \pm 0.05$ \\
\hline & 000622.8 & +201458.0 & UVW2 & & $17.68 \pm 0.20$ & $17.73 \pm 0.04$ & $17.78 \pm 0.03$ & $17.81 \pm 0.03$ \\
\hline & 000632.0 & +201308.3 & $U$ & $16.53 \pm 0.02$ & $16.54 \pm 0.02$ & $16.55 \pm 0.03$ & $16.57 \pm 0.02$ & $16.55 \pm 0.02$ \\
\hline & 000632.0 & +201308.3 & UVW1 & $\ldots$ & $17.26 \pm 0.03$ & $18.30 \pm 0.07$ & $18.20 \pm 0.05$ & $18.17 \pm 0.05$ \\
\hline & 000632.0 & +201308.3 & UVM2 & $\ldots$ & $\ldots$ & $>20.03$ & $>20.35$ & $>20.42$ \\
\hline & 000632.0 & +201308.3 & UVW2 & $\ldots$ & $\ldots$ & $19.76 \pm 0.12$ & $19.76 \pm 0.10$ & $19.91 \pm 0.10$ \\
\hline \multirow[t]{3}{*}{ 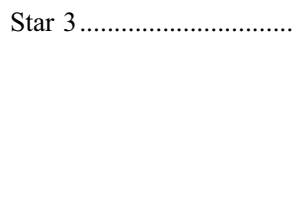 } & 000617.1 & +201408.4 & V & $15.40 \pm 0.02$ & $\ldots$ & $15.46 \pm 0.02$ & $15.43 \pm 0.02$ & $15.42 \pm 0.01$ \\
\hline & 000617.1 & +201408.4 & $B$ & $16.21 \pm 0.01$ & $16.19 \pm 0.01$ & $16.13 \pm 0.02$ & $16.13 \pm 0.01$ & $16.09 \pm 0.01$ \\
\hline & 000617.1 & +201408.4 & $U$ & $16.27 \pm 0.02$ & $16.26 \pm 0.02$ & $16.26 \pm 0.02$ & $16.26 \pm 0.02$ & $16.23 \pm 0.02$ \\
\hline & 000618.0 & $+2013 \quad 17.2$ & $B$ & $15.51 \pm 0.01$ & $15.49 \pm 0.02$ & $15.46 \pm 0.01$ & $15.45 \pm 0.01$ & $15.44 \pm 0.01$ \\
\hline & 000618.0 & $+2013 \quad 17.2$ & $U$ & $15.35 \pm 0.01$ & $15.36 \pm 0.01$ & $15.33 \pm 0.01$ & $15.35 \pm 0.01$ & $15.34 \pm 0.01$ \\
\hline & 000618.0 & $+2013 \quad 17.2$ & UVW1 & $\ldots$ & $15.70 \pm 0.02$ & $16.35 \pm 0.02$ & $16.34 \pm 0.02$ & $16.37 \pm 0.02$ \\
\hline & 000618.0 & +2013 17.2 & UVM2 & $16.85 \pm 0.12$ & $16.77 \pm 0.06$ & $16.99 \pm 0.04$ & $17.02 \pm 0.03$ & $17.09 \pm 0.03$ \\
\hline & 000618.0 & +2013 17.2 & UVW2 & & $17.16 \pm 0.13$ & $17.35 \pm 0.03$ & $17.37 \pm 0.02$ & $17.42 \pm 0.02$ \\
\hline \multirow[t]{6}{*}{ Star $5 \ldots \ldots \ldots \ldots \ldots \ldots \ldots$} & 000620.1 & +201050.2 & V & $14.30 \pm 0.01$ & $\ldots$ & $14.31 \pm 0.01$ & $14.33 \pm 0.01$ & $14.30 \pm 0.01$ \\
\hline & 000620.1 & +201050.2 & $B$ & $15.51 \pm 0.01$ & $15.47 \pm 0.01$ & $15.38 \pm 0.01$ & $15.39 \pm 0.01$ & $15.39 \pm 0.01$ \\
\hline & 000620.1 & +201050.2 & $U$ & $16.45 \pm 0.02$ & $16.45 \pm 0.02$ & $16.46 \pm 0.03$ & $16.41 \pm 0.02$ & $16.48 \pm 0.02$ \\
\hline & 000620.1 & +201050.2 & UVW1 & $\ldots$ & $17.54 \pm 0.04$ & $18.01 \pm 0.06$ & $18.12 \pm 0.05$ & $18.12 \pm 0.04$ \\
\hline & 000620.1 & +201050.2 & UVM2 & $\ldots$ & $\ldots$ & $>20.03$ & $>20.34$ & $>20.39$ \\
\hline & 000620.1 & +201050.2 & UVW2 & $\ldots$ & $\ldots$ & $19.22 \pm 0.09$ & $19.44 \pm 0.08$ & $19.52 \pm 0.07$ \\
\hline
\end{tabular}

NотеS.- Units of right ascension are hours, minutes, and seconds, and units of declination are degrees, arcminutes, and arcseconds. The magnitudes are not corrected for Galactic reddening.

${ }^{a}$ The observing times of the 2007 Swift observations are listed in Table 1 of Grupe et al. (2007a).

to determine when and how fast this recent flux drop occurred, the RXTE ASM light curve was examined. The month-to-month ASM light curve indicates that Mrk 335 has been variable in $\mathrm{X}$-rays over the last decade. However, Mrk 335 is too faint to constrain the occurrence of the most recent flux drop with the ASM. Mrk 335 also appears to be fainter in the UV in 2007 compared with previous epochs. Table 4 lists the XMM-Newton OM and Swift UVOT measurements. During the 2007 May 17 Swift observation, Mrk 335 appears to be fainter in the UV by about 0.2 mag compared to the Swift and XMM-Newton observations before and after.

\subsubsection{Short-Term Variability}

The left panel of Figure 2 shows the 2007 July XMM-Newton EPIC pn light curve in the $0.2-10.0 \mathrm{keV}$ band. During the $16.8 \mathrm{ks}$ exposure the AGN showed a steady decay in flux by about $15 \%$. The light curve is relatively quiescent compared to the high flux state variability seen in the 2006 XMM-Newton and Suzaku light curves (O’Neill et al. 2007; Larsson et al. 2008, respectively). The variability is in agreement with the variability-flux dependence reported by Uttley \& McHardy (2001). However, the 2007 July XMM-Newton observation was rather short (17 ks). During the low-state Swift observation on 2007 May 25, which covers a time period of $70 \mathrm{ks}$, Mrk 335 is quite variable by a factor of about 2 within $20 \mathrm{ks}$ (Fig. 2, right panel). In both cases the AGN is variable and deviates significantly from a constant value. The $\chi^{2}$ tests show that the $\chi^{2} / \nu$ is $49 / 15$ and $55 / 11$ for the $X M M$ Newton pn and Swift XRT light curves, respectively, assuming the mean count rates as constant values.

In Figure 3 the XRT and UVW2 light curves for all Swift segments between 2007 May 17 and July 2 are displayed (the magnitudes of segments 001-005 are listed in Table 4). The light curves show that Mrk 335 is quite variable on intermediate timescales of days to weeks. Note that the UVW2 light curve follows the XRT count rate, suggesting some connection between the optical/UV and X-ray variability. The hardness $\mathrm{ratio}^{7}$ variability curve may suggest some spectral variability in the X-ray band (Fig. 3, middle panel); however, a $\chi^{2}$ test shows that this is not significant considering the uncertainties.

\subsection{X-Ray Spectral Analysis}

Table 5 summarizes the results from the X-ray spectral analysis in the $0.3-12.0 \mathrm{keV}$ range of the 2007 XMM-Newton ToO (low flux state) and 2006 XMM-Newton GO (high flux state) observations. The 2007 EPIC pn and MOS spectra cannot be fitted by a single absorbed power-law model. This is similar to the previous XMM-Newton and Swift observations (Grupe et al. 2007a), which cannot be fitted by a single power-law model either. Applying a broken power-law model to the pn and MOS data

\footnotetext{
7 The hardness ratio is defined as HR $=(H-S) /(H+S)$, where $S$ and $H$ are the number of photons in the $0.3-1.0 \mathrm{keV}$ and $1.0-10.0 \mathrm{keV}$ bands, respectively.
} 


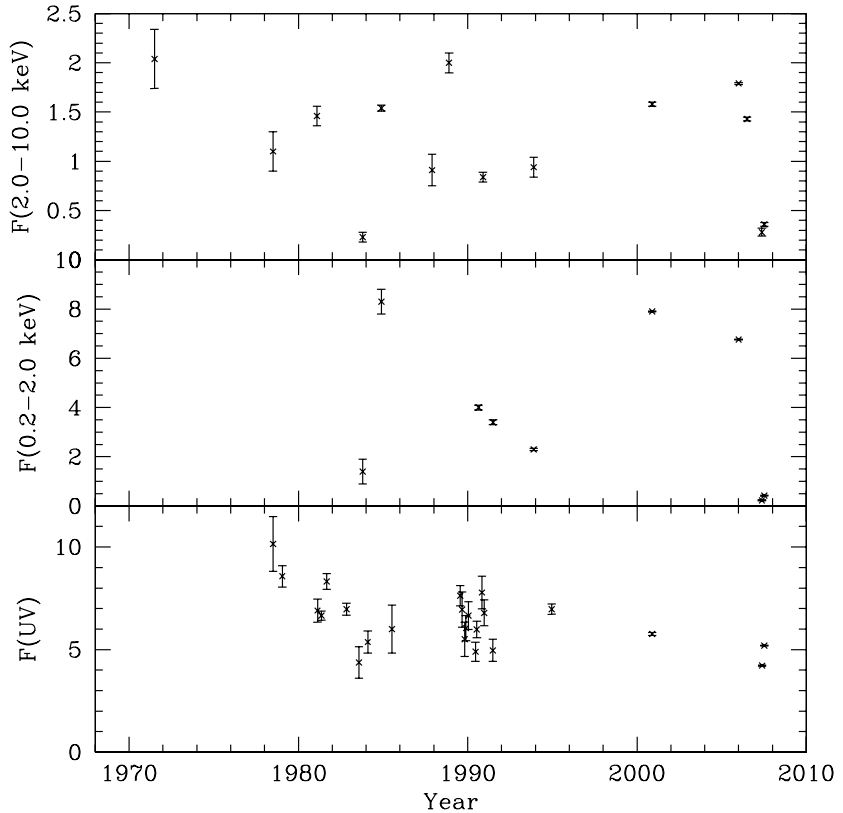

Fig. 1.- Long-term light curves of Mrk 335. The top panel shows the 2$10 \mathrm{keV}$ light curve starting with the first X-ray detection of Mrk 335 by Uhuru in 1971. The middle panel displays the $0.2-2.0 \mathrm{keV}$ flux light curve starting at the EXOSAT observation in 1983 November. The bottom panel displays the UV flux light curve measured with IUE and HST at $2256 \AA$ and XMM-Newton $\mathrm{OM}$ and Swift UVOT using the M2 filters. Note that these UV data are not corrected for Galactic reddening. The XMM-Newton data are adjusted to the Swift UVOT data. All fluxes are given in units of $10^{-14} \mathrm{~W} \mathrm{~m}^{-2}\left(=10^{-11} \mathrm{ergs} \mathrm{s}^{-1} \mathrm{~cm}^{-2}\right)$ as listed in Table 3.

significantly improves the fit, but as shown in Figure 4, the X-ray spectrum during the $2007 X M M-N e w t o n$ ToO observation is quite complicated and requires further components. There are strong residuals at energies below $1 \mathrm{keV}$ and between 3 and $7 \mathrm{keV}$. The residuals at lower energies can be associated with strong X-ray emission lines found in the RGS spectra of Mrk 335 (see $\S 3.2 .4$ ). Figure 4 also shows that the pn and MOS data are consistent with each other. Due to some internal processing problems, MOS data were not made available to us in time to be handled in the full analysis. In order to represent soft X-ray emission lines, we added two Gaussian profiles at 0.5 and $0.9 \mathrm{keV}$. These Gaussians do not represent specific lines but are used to mimic line emission and improve the fit. The emission feature at $6.4 \mathrm{keV}$ is associated with near-neutral $\mathrm{Fe} \mathrm{K} \alpha$ emission (e.g., Fe I-XVII) and is modeled with a single, narrow Gaussian. The addition of these three Gaussian profiles to the broken power-law model improves the fit, but significant deviations remain in the $2-6 \mathrm{keV}$ range. These residuals can be improved with an extremely broad Gaussian with a peak at $E=4.9 \mathrm{keV}$, a width $\sigma=1.2 \mathrm{keV}$, and an equivalent width $\mathrm{EW}=2.4 \mathrm{keV}$. (Fig. 5). Even though this yields an acceptable fit, the line widths and equivalent width seem to be unusually extreme for a broad line (Guainazzi et al. 2006), although the presence of such a line was suggested in earlier observations (Gondoin et al. 2002; Longinotti et al. 2007b). This additional component is not necessary when a partial covering model is applied to the data.

The narrow $6.4 \mathrm{keV}$ Fe line is present in the high- and low-flux spectra. The fluxes in the $\mathrm{Fe} \mathrm{K} \alpha 6.4 \mathrm{keV}$ line in the 2006 and 2007 XMM-Newton observations are $(1.83 \pm 0.33) \times 10^{-16}$ and $(1.15 \pm 0.45) \times 10^{-16} \mathrm{~W} \mathrm{~m}^{-2}$, respectively. This result suggests that the flux in the $\mathrm{Fe} \mathrm{K} \alpha 6.4$ line has become fainter in the low $\mathrm{X}$-ray flux state compared to the high state. The equivalent widths (EWs), however, are different due to the much lower continuum flux during the 2007 observation. We found $\mathrm{EW}=100 \pm 20$ and $200 \pm 80 \mathrm{eV}$ during the 2006 and 2007 observations, respectively. While the $6.4 \mathrm{keV} \mathrm{Fe} \mathrm{K} \alpha$ line is clearly present in both spectra, the $7.0 \mathrm{keV}$ Fe XXvi Ly $\alpha$ line that was found in the 2006 $X M M-N e w t o n$ observation (O'Neill et al. 2007) is absent during the low state in 2007.

Figure 6 displays the difference spectrum between the 2006 high state and 2007 low state fitted in the $2-5 \mathrm{keV}$ energy range with a Galactic absorbed power law $\left(\alpha_{\mathrm{X}}=1.3\right)$ and then extrapolated to lower and higher energies. This difference spectrum clearly shows that the changes between the high and the low state happened primarily at soft energies below $1.5 \mathrm{keV}$. In some ways this plot is similar to the high- and low-state SEDs shown in Figure 2 of Grupe et al. (2007b). Obviously the high- and lowstate spectra cannot be described simply by a renormalization of a single power-law model. Despite the fact that most of the flux

TABLE 3

Summary of the $0.2-2.0 \mathrm{keV}$ and $2.0-10.0 \mathrm{keV}$ Fluxes

\begin{tabular}{|c|c|c|c|c|}
\hline Mission & Observing Time & $0.2-2.0 \mathrm{keV}$ Flux ${ }^{\mathrm{a}}$ & $2.0-10.0 \mathrm{keV}$ Flux $^{\mathrm{a}}$ & References \\
\hline 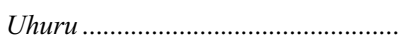 & $1971 / 1972$ & $\ldots$ & $2.04 \pm 0.03$ & 1 \\
\hline 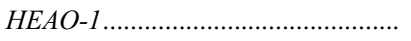 & 1978 Jul 7 & $\ldots$ & $1.1 \pm 0.2$ & 1 \\
\hline 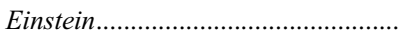 & 1981 Jan & $\ldots$ & $1.46 \pm 0.10$ & 1 \\
\hline 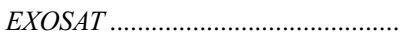 & 1983 Nov 5 & $1.4 \pm 0.2$ & $0.23 \pm 0,05$ & 2 \\
\hline 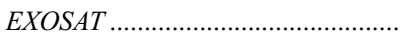 & 1984 Dec 6 & $8.3 \pm 0.1$ & $1.54 \pm 0.03$ & 2 \\
\hline Ginga & 1987 Dec 3 & $\ldots$ & $0.91 \pm 0.16$ & 3 \\
\hline Ginga & 1988 Nov 29 & $\ldots$ & $2.00 \pm 0.10$ & 3 \\
\hline ROSAT RASS & 1990 Jul 14 & $4.00 \pm 0.10$ & $\ldots$ & 4 \\
\hline 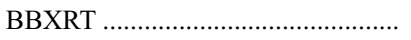 & 1990 Dec 5 & & $0.84 \pm 0.05$ & 1 \\
\hline ROSAT PSPC & 1991 Jun 30 & $3.40 \pm 0.10$ & $\ldots$ & 4 \\
\hline$A S C A$ & 1993 Dec 9 & $\ldots$ & $0.94 \pm 0.10$ & 5 \\
\hline 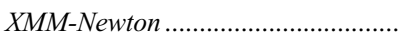 & 2000 Dec 25 & $7.90 \pm 0.01$ & $1.58 \pm 0.02$ & 6 \\
\hline 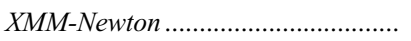 & 2006 Jan 3 & $6.76 \pm 0.01$ & $1.79 \pm 0.01$ & 6 \\
\hline 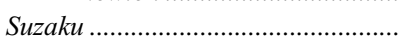 & 2006 Jun 20 & $\ldots$ & $1.43 \pm 0.02$ & 7 \\
\hline 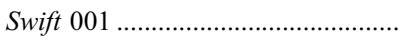 & 2007 May 17 & $0.23 \pm 0.03$ & $0.28 \pm 0.04$ & 6 \\
\hline XMM-Newton & 2007 Jul 10 & $0.41 \pm 0.01$ & $0.31 \pm 0.01$ & 8 \\
\hline
\end{tabular}

a The $0.2-2.0$ and $2.0-10.0 \mathrm{keV}$ fluxes are corrected for Galactic absorption and are given in units of $10^{-14} \mathrm{~W} \mathrm{~m}^{-2}\left(=10^{-11} \mathrm{ergs} \mathrm{s}^{-1} \mathrm{~cm}^{-2}\right)$. References.-(1) Turner et al. 1993; (2) Pounds et al. 1987; (3) Nandra \& Pounds 1994; (4) Grupe et al. 2001; (5) George et al. 2000; Leighly 1999a; (6) Grupe et al. 2007a; (7) Larsson et al. 2008; (8) this paper. 
TABLE 4

Optical and UV Photometry of Mrk 335 Using the Swift UVOT and XMM-Newton OM

\begin{tabular}{|c|c|c|c|c|c|c|c|}
\hline Filter & XMM 2000 & XMM 2007 & Swift 001 & Swift 002 & Swift 003 & Swift 004 & Swift 005 \\
\hline$V \ldots \ldots \ldots$ & $14.00 \pm 0.01$ & & $14.22 \pm 0.01$ & $14.19 \pm 0.01$ & $14.19 \pm 0.01$ & $14.15 \pm 0.01$ & $14.18 \pm 0.01$ \\
\hline 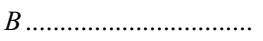 & $14.15 \pm 0.01$ & $14.25 \pm 0.01$ & $14.49 \pm 0.01$ & $14.42 \pm 0.01$ & $14.44 \pm 0.01$ & $14.41 \pm 0.01$ & $14.42 \pm 0.01$ \\
\hline ....................... & $13.00 \pm 0.01$ & $13.17 \pm 0.01$ & $13.29 \pm 0.01$ & $13.20 \pm 0.01$ & $13.23 \pm 0.01$ & $13.22 \pm 0.01$ & $13.23 \pm 0.01$ \\
\hline 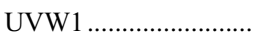 & & $13.52 \pm 0.01$ & $13.20 \pm 0.01$ & $13.08 \pm 0.02$ & $13.09 \pm 0.02$ & $13.10 \pm 0.01$ & $13.05 \pm 0.01$ \\
\hline 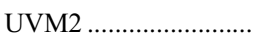 & $12.92 \pm 0.16$ & $12.90 \pm 0.01$ & $13.12 \pm 0.01$ & $12.95 \pm 0.01$ & $12.98 \pm 0.01$ & $12.97 \pm 0.01$ & $12.93 \pm 0.01$ \\
\hline 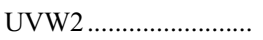 & $\ldots$ & $13.07 \pm 0.20$ & $13.19 \pm 0.01$ & $13.01 \pm 0.01$ & $13.04 \pm 0.01$ & $13.04 \pm 0.01$ & $12.98 \pm 0.01$ \\
\hline
\end{tabular}

NotE.-All magnitudes are corrected for Galactic reddening $\left(E_{B-V}=0.035\right.$; Schlegel et al. 1998), and the XMM-Newton OM magnitudes are adjusted as described in the text to be comparable with the Swift UVOT data.

change between 2006 and 2007 occurs at lower energies, the soft X-ray spectral slopes in the 2006 and 2007 XMM-Newton observations are similar. From a broken power-law model fit the soft X-ray spectral slopes of the 2006 high and 2007 low states are $\alpha_{\mathrm{X}}=1.73 \pm 0.01$ and $1.89 \pm 0.04$, respectively. At higher energies, however, the energy spectral indices are significantly different. During the 2006 XMM-Newton observation, the slope was about $\alpha_{\mathrm{X}}=1.0$, which is quite typical for an NLS1 (e.g., Leighly 1999a). The spectral slope in the 2007 XMM-Newton observation is very hard with $\alpha_{\mathrm{X}}=-0.1$. We investigate two models that can explain such a spectral shape: a partial covering absorber and reflection from an ionized accretion disk.

\subsubsection{The Partial Covering Absorber Scenario}

Partially covering a single power-law continuum significantly improves the fit to the low-flux 2007 spectrum compared to the broken power-law model. The models still require three Gaussian profiles to fit the low-energy emission features and the narrow $6.4 \mathrm{keV}$ emission line $\left(\chi^{2} / \nu=380 / 299\right)$. The broad Gaussian profile around $5 \mathrm{keV}$ used in the broken power-law model is not required. In this scenario the intrinsic power law has an energy spectral slope $\alpha_{\mathrm{X}}=1.78$. The absorber has a column density of $N_{\mathrm{H}}=15.1 \times 10^{22} \mathrm{~cm}^{-2}$ and a covering fraction $f_{c}=0.94$. These partial covering parameters are comparable to the ones found by Swift during the 2007 May and June observations (Grupe et al. $2007 \mathrm{~b})$. The partial covering parameters for the 2006 observation are then $N_{\mathrm{H}, \mathrm{pc}}=(5.5 \pm 0.2) \times 10^{22} \mathrm{~cm}^{-2}$ and $f_{c}=0.32 \pm 0.01$.

Even though a comparison of the 2007 XMM-Newton data with the 2007 May and June Swift observations suggests some variability in the column density, the spectral parameters are consistent during these low-flux periods. Notably, all spectra fitted by the partial covering absorber model only require a single underlying power-law spectrum. The spectral slope during all these low-state observations remains fairly constant within uncertainties at $\alpha_{\mathrm{X}}=1.8$. The column densities were always within a factor of 2 . The covering fraction at all four epochs was $f_{c}=$ 0.94 . The column density was highest during the 2007 May 17 Swift observation with $N_{\mathrm{H}, \mathrm{pc}}=2 \times 10^{23} \mathrm{~cm}^{-2}$ and lowest on May 25 with $N_{\mathrm{H}, \mathrm{pc}}=1 \times 10^{23} \mathrm{~cm}^{-2}$ (Grupe et al. 2007a). The column densities during the 2007 June Swift and 2007 July 10 XMMNewton observations are in between those values with $N_{\mathrm{H}, \mathrm{pc}}=$ $1.5 \times 10^{23} \mathrm{~cm}^{-2}$. The covering fraction during all four observations was $f_{c}=0.94$.

One way to test whether the partial covering absorber model is self-consistent, we fitted the 2006 and 2007 pn data simultaneously in XSPEC. This test can show if the same underlying continuum spectrum can be applied to the data only affected by the absorber column density and covering fraction. The first test was to use a model with two power laws (replacing the broken power law) and one partial covering absorber. While the power-law parameters were tied, the partial covering absorber parameters were left free to vary, first only the covering fraction and second also the absorber column density. In none of these cases could we obtain an acceptable fit to the spectra. This result suggests that high and low states have different underlying continua. The situation changes when adding an additional partial covering absorber to the model [XSPEC model wabs*zpcfabs*zpcfabs*( powl+ powl)]. This model significantly improved the fit from a reduced $\chi^{2}=$ 2.67 using the one partial covering absorber to $\chi^{2}=1.58$ with the two partial covering absorbers. One of the partial covering absorber components, however, requires a column density of $3 \times 10^{24} \mathrm{~cm}^{-2}$. The covering fractions, which were left as a free parameter, of this component are $f_{c}=0.2$ and 0.8 for the high and low states, respectively.

As mentioned by Grupe et al. (2007a), when correcting the fluxes during the low-state observations for Galactic and intrinsic
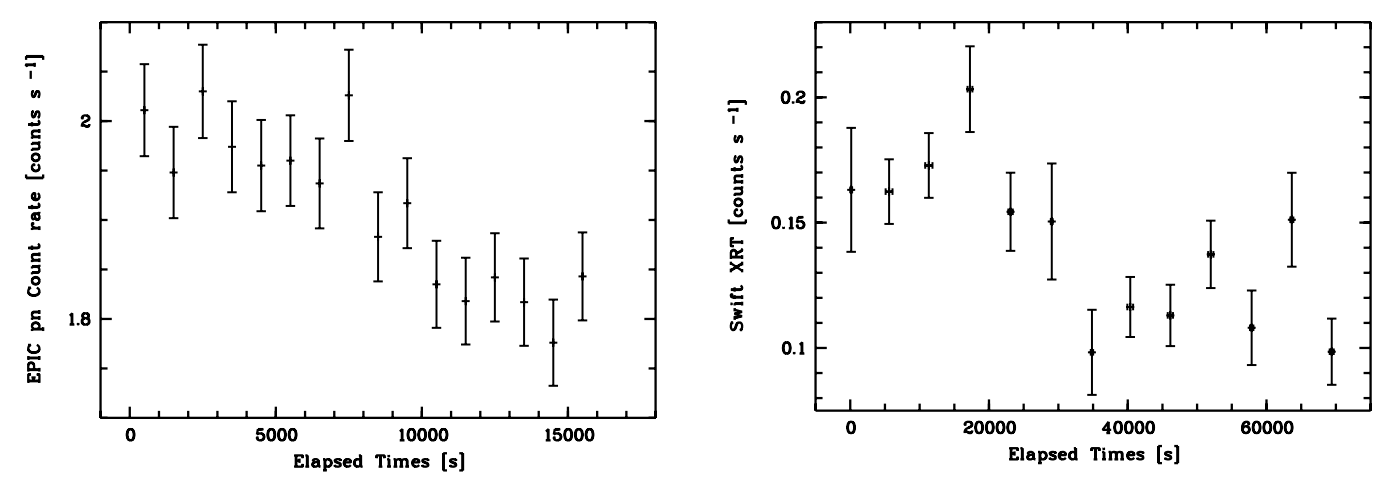

FIG. 2.-2007 XMM-Newton EPIC pn light curve in the 0.2-10.0 keV band (left) and Swift XRT 0.3-10.0 keV light curve of the second Swift observation on 2007 May 25 (Grupe et al. 2007a; right). The starting times of the observations are 2007 July 10 17:09 UT and 2007 May 25 00:01 UT, respectively. 


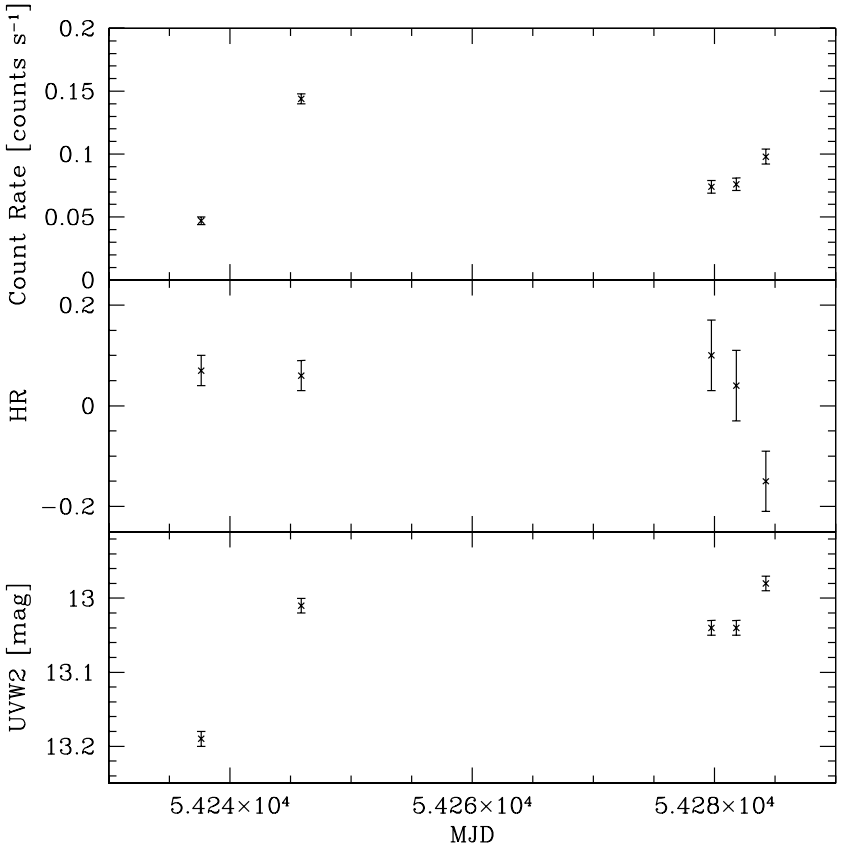

FIG. 3.-Swift XRT and UVOT UVW2 light curves of Mrk 335 between 2007 May 17 and June 2 . The top panel shows the XRT count rate, the middle panel the XRT hardness ratio (see $\S 3.1 .2$ ), and the bottom panel the UVOT UVW2 light curve.

absorption, the intrinsic fluxes are similar and the intrinsic longterm X-ray flux amplitude varies by a factor of about 4-6 (Grupe et al. 2007a), which is similar to other NLS1 galaxies (e.g., Grupe et al. 2001). The unabsorbed fluxes in the $0.2-2.0 \mathrm{keV}$ and 2.0-10.0 keV bands during the 2006 XMM-Newton obser- vation were $6.7 \times 10^{-14}$ and $6.4 \times 10^{-14} \mathrm{~W} \mathrm{~m}^{-2}$, respectively, which is very similar to the low-state $X M M$-Newton observations on 2007 July 10 , which were $6.9 \times 10^{-14}$ and $5.4 \times 10^{-14} \mathrm{~W} \mathrm{~m}^{-2}$, respectively. Note that during the extreme low state on 2007 May 17 observed by Swift, the fluxes were $4.1 \times 10^{-14}$ and $3.2 \times$ $10^{-14} \mathrm{~W} \mathrm{~m}^{-2}$, respectively. These values do not suggest significant intrinsic flux variability. Note that the absorption-corrected rest-frame 0.2-2.0 keV flux during the ROSAT All Sky Survey (RASS; Voges et al. 1999) was $4 \times 10^{-14} \mathrm{~W} \mathrm{~m}^{-2}$ (Table 3), very similar to the flux found during the 2007 May 17 Swift observations.

\subsubsection{A Reflection Interpretation for Mrk 335}

The 2007 low-flux, broadband X-ray spectrum of Mrk 335 can be well fitted with a single power-law continuum and blurred ionized reflection $\left(\chi_{\nu}^{2}=1.1\right)$. It has been shown that in some NLS1s the same low-flux model can be applied to the high flux state by simply renormalizing the power-law component (e.g., Fabian et al. 2004; Gallo et al. 2007a). However, this is not the case for Mrk 335 as renormalizing the power law results in a poor fit to the 2006 high flux state. Allowing both the power-law and reflection components to vary freely cannot simultaneously describe the high-energy $(E=2-10 \mathrm{keV})$ and low-energy $(E<$ $2 \mathrm{keV}$ ) spectra of Mrk 335 during the high flux state. Similar difficulties were encountered by O'Neill et al. (2007) with the same data set and demonstrate the need for an additional continuum component during the high state.

One possibility proposed to describe the flaring in I Zw 1 (Gallo et al. 2007a, 2007b) was that different parts of the accretion disk are illuminated by different continuum spectra. To model this scenario, we introduce a second power law with (model A) and without (model B) associated reflection. In model A we assume that a steep power law (with photon index $\Gamma_{1}$ ) illuminates the

TABLE 5

Spectral Analysis of the 2007 and 2006 XMM-Newton Spectra of Mrk 335 in the 0.3-12.0 keV Range

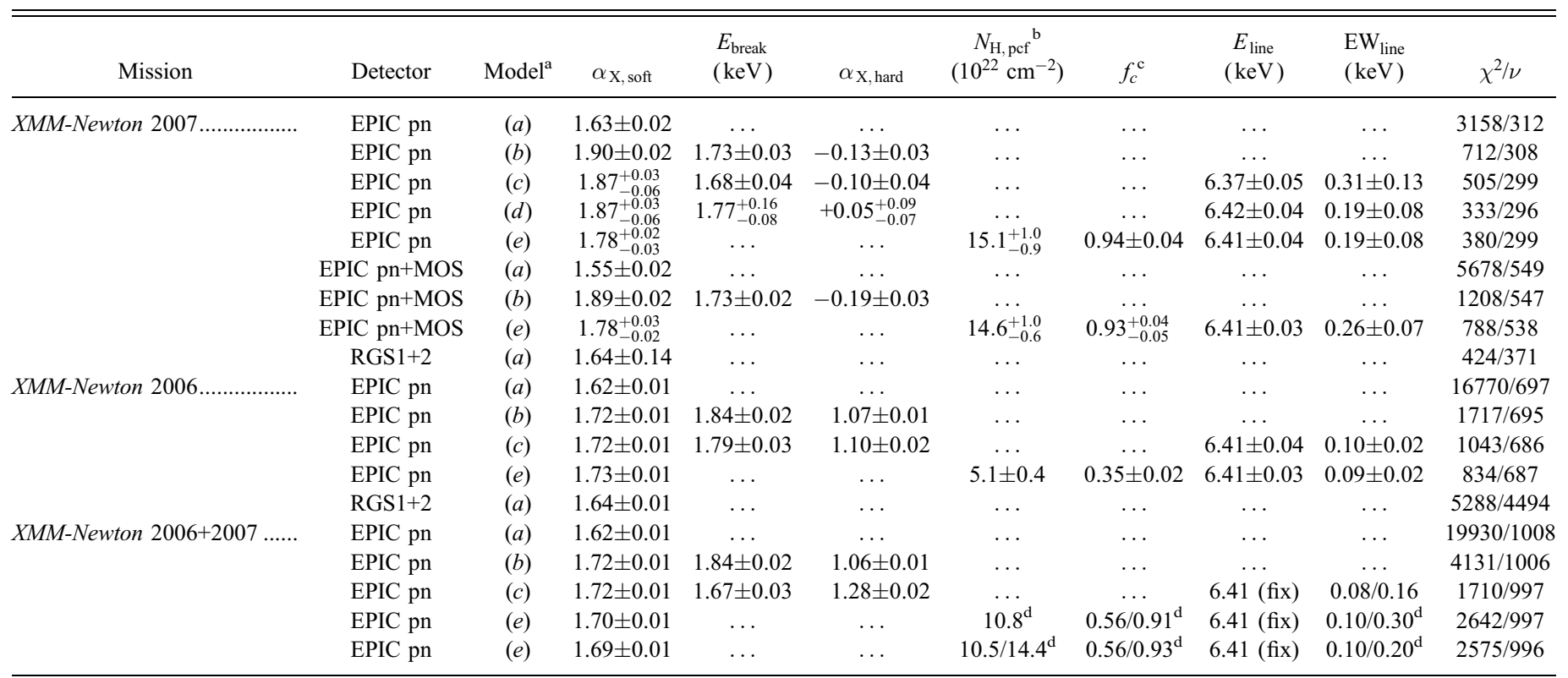

${ }^{a}$ Spectral models used are $(a)$ absorbed power law, $(b)$ absorbed broken power law, $(c)$ absorbed broken power law with two Gaussians to represent the soft X-ray emission lines below $1 \mathrm{keV}$ and one Gaussian for the $6.4 \mathrm{keV} \mathrm{Fe} \mathrm{line,}(d)$ same as $(c)$ but with an additional broad line at $4.9 \mathrm{keV}$ with $\sigma=1.5 \mathrm{keV}$, and $(e)$ power law with a partial covering absorber with two soft X-ray Gaussians and one for the $6.4 \mathrm{Fe}$ line. For all models the absorption column density of the $z=0$ absorber was fixed to the Galactic value $\left(3.96 \times 10^{20} \mathrm{~cm}^{-2}\right.$; Dickey \& Lockman 1990).

${ }^{\mathrm{b}}$ Absorption column density of the partial covering absorber $N_{\mathrm{H}, \mathrm{pcf}}$.

${ }^{\mathrm{c}}$ Covering fraction.

d Simultaneous fit with a power law with partial covering absorber and Gaussian lines. While the X-ray spectral slope was tied and the line energies and widths were fixed, the column densities and covering fractions of the partial covering absorber and the normalizations of the power-law continua and the line fluxes were left as free parameters. The values of the column densities, covering fractions, and equivalent widths of the $6.4 \mathrm{keV}$ line are given for the 2006 and 2007 data. 


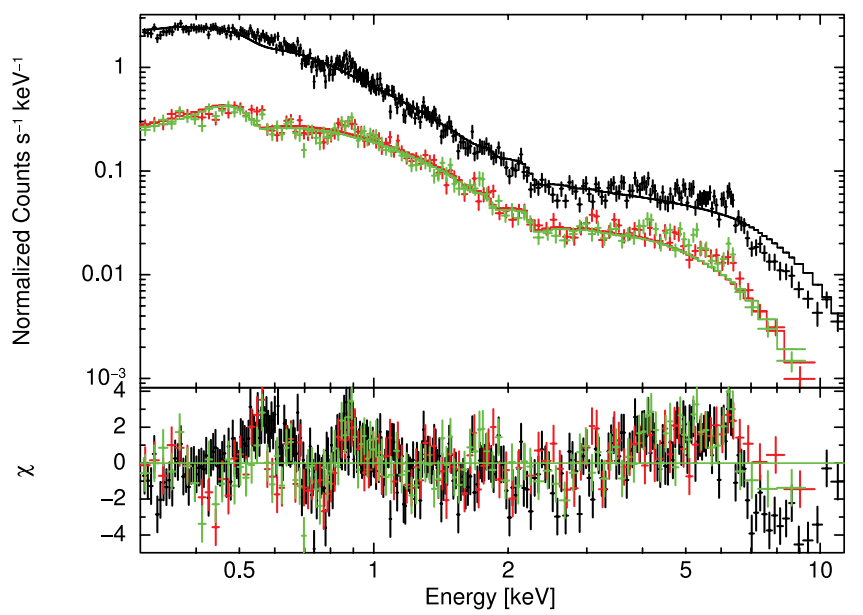

FIG. 4.-XMM-Newton EPIC pn 0.3-12.0 keV (black) and MOS 0.3$10.0 \mathrm{keV}$ (red + green) spectra of Mrk 335 fitted with a broken power law with $\alpha_{\mathrm{X} \text {,soft }}=1.89, \alpha_{\mathrm{X} \text {,hard }}=-0.19, E_{\text {break }}=1.73 \mathrm{keV}$ as listed in Table 5 . The absorption column density is fixed to the Galactic value $\left(3.96 \times 10^{20} \mathrm{~cm}^{-2}\right.$; Dickey \& Lockman 1990).

inner accretion disk from $r_{\text {in }}$ to $r_{\text {br }}$, while a flatter power law $\left(\Gamma_{2}\right)$ is associated with outer disk reflection from $r_{\text {br }}$ to $r_{\text {out }}$. In model B, no reflection is associated with the steep power law, which may be explained if that component is beamed away from the disk; on the other hand, in model B we assume a broken power-law emissivity profile for the single reflector with an inner emissivity index $q_{\text {in }}$, breaking to $q_{\text {out }}$ at $r_{\text {br }}$. The two different continuum slopes may in fact be due to different mechanisms (e.g., bulk motion Comptonization and standard thermal Comptonization; see, e.g., Niedzwiecki \& Zdziarski 2006).

In addition, a MEKAL hot diffuse gas component with $\mathrm{Fe}$ abundance tied to that of the reflector(s) is included to account for any distant emission due to thermal plasma, which should account for the ionized Fe L lines tentatively detected in the RGS during the lower flux state. The parameters of this component are linked in all observations. As with any other model discussed above, we also include a narrow $\mathrm{Fe} \mathrm{K} \alpha$ emission line at $\sim 6.4 \mathrm{keV}$ and a $\sim 7 \mathrm{keV}$ line, which is, however, significantly detected only in the high flux state 2006 XMM-Newton observation. A narrow $\mathrm{Ne}$ line with constant intensity at $\sim 0.9 \mathrm{keV}$ is also included in all fits.

Both model A and model B describe the various flux states well (Fig. 7). See Table 6 for fit parameters. In both models the difference between the high and low flux state is primarily attrib- uted to the significantly diminished flux of the steeper power-law component. While the two models differ in their interpretation of the second power law (e.g., beamed vs. unbeamed continuum emission), these are issues that can be tested with further analysis (L. C. Gallo et al. 2008, in preparation). Both models are successful in describing the spectra, but they both require additional components to explain the soft excess.

\subsubsection{Hard X-Ray BAT Spectrum}

Mrk 335 is detected in the 22 month Swift Burst Alert Telescope (BAT; Barthelmy et al. 2005) AGN survey (Tueller et al. 2008). The BAT spectrum can be fitted by a single power law with $\alpha_{\mathrm{X}}=1.77_{-0.64}^{+0.87}$. This is a similar slope as found in the Swift XRT and XMM-Newton low-state data fitted with a single power law and partial covering absorber. Figure 8 displays the $X M M$ Newton pn and Swift BAT spectra fitted simultaneously in XSPEC with a power law with a partial covering absorber with $\alpha_{\mathrm{X}}=$ $1.85 \pm 0.03, N_{\mathrm{H}, \mathrm{pc}}=(16.4 \pm 1.0) \times 10^{22} \mathrm{~cm}^{-2}$, and a covering fraction of $f_{c}=0.94 \pm 0.01\left(\chi^{2} / \nu=582 / 315\right)$. The normalizations of the power-law models were left free. Note that the BAT spectrum is the average spectrum over 22 months, so it is not simultaneous with the XMM-Newton low-state spectrum. The observed 20-40 keV and 20-100 keV fluxes are $7.6 \times 10^{-15}$ and $1.33 \times 10^{-14} \mathrm{~W} \mathrm{~m}^{-2}$, respectively, which is in agreement with the measurements from the 2006 June Suzaku observation (Larsson et al. 2008). We do not know from the BAT data if the flux in the high-energy bands was lower during the low state in 2007 MayJuly. However, the AGNs in the 22 month survey do not show strong variability compared with what is typically observed at lower energies (J. Tueller 2008, private communication).

\subsubsection{Soft X-Ray Emission Lines in the RGS}

The X-ray slopes obtained from single power-law fits to the EPIC pn (0.2-2.0 keV) and RGS (0.35-2.2 keV) spectra are consistent $\left(\alpha_{\mathrm{X}}=1.7\right)$ for the 2006 and 2007 observations.

The low-flux, broadband X-ray spectra of Mrk 335 in Figure 4 show significant residuals below $2 \mathrm{keV}$ when fitted with a single power law. The moderate-resolution spectrum suggests the presence of an ionized gas in either absorption or emission, or both. Figure 9 shows the higher resolution RGS spectrum of Mrk 335 fitted with a single power-law model. The residuals from this fit clearly show the presence of strong emission lines around 13, 19, 22 , and $25 \AA$.

The combined RGS1 and RGS2 spectrum is shown in the lower four panels of Figure 9 in the wavelengths bands 7-14 $\AA$, 13-21 $\AA, 20-28 \AA$, and 27-34 $\AA$. Possible identification of the most likely features is reported. Most of these lines seem to be
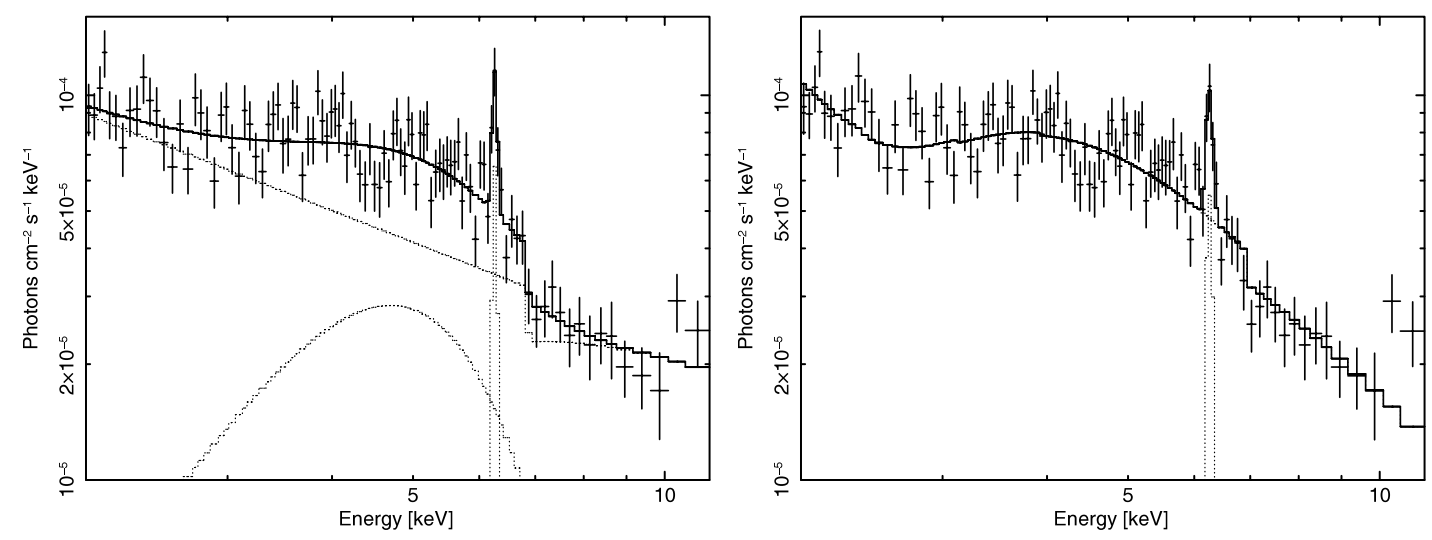

Fig. 5. - XMM-Newton EPIC pn 2.0-12.0 keV spectrum of Mrk 335 with a power law and a broad Fe line at $4.9 \mathrm{keV}$ (left) and a power law with partial covering absorber (right). 


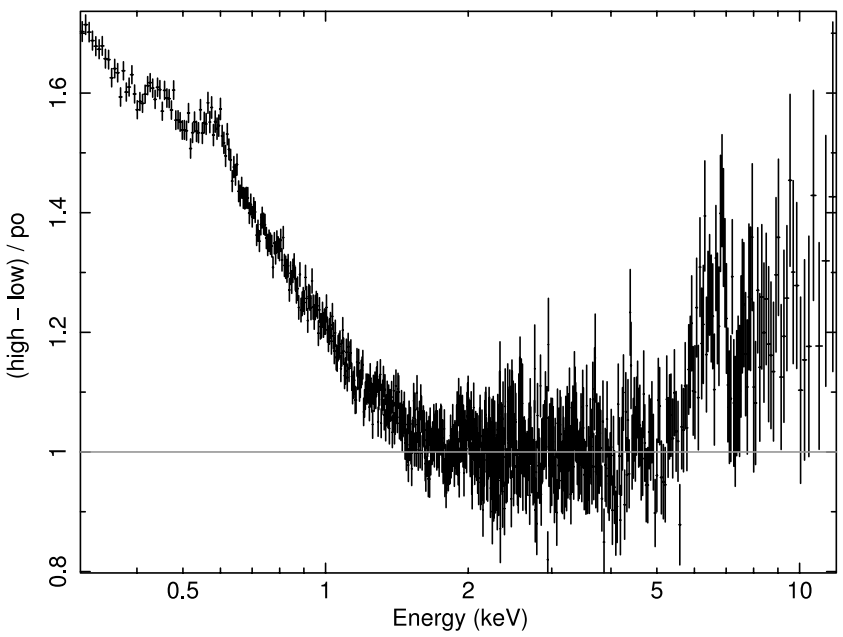

FIG. 6.-Difference spectrum between the 2006 high-state and 2007 low-state $X M M-N e w t o n$ spectra fitted in the $2-5 \mathrm{keV}$ range by a Galactic absorber power law with $\alpha_{\mathrm{X}}=1.3$ and extrapolated to lower and higher energies. [See the electronic edition of the Journal for a color version of this figure.]

highly ionized Fe lines including some Fe group elements like $\mathrm{Mn}$ or Ni. We could also identify Ne IX at $13 \AA$. This line, however, is not as strong and prominent as was found in the spectrum of the partial covering candidate and highly optically polarized NLS1 Mrk 1239 (Grupe et al. 2004b). The most prominent group of lines that we have identified is the helium $\mathrm{K} \alpha$ complex of $\mathrm{O}$ VII emission lines around $22 \AA$. These lines can potentially be used for line diagnostics to measure properties of the line-emitting plasma $(\S 4.2)$.

\subsection{UV/Optical Data}

\subsubsection{UVOT and OM Photometry}

The UV/optical photometric measurements of Mrk 335 made with the XMM-Newton OM and Swift UVOT are listed in Table 4. The values given in the table are corrected for Galactic reddening $\left(E_{B-V}=0.035\right.$; Schlegel et al. 1998), and the OM magnitudes were adjusted, as described in $\S 2$, to be comparable with the Swift UVOT magnitudes. The data suggest that Mrk 335 was slightly brighter in the optical/UV in 2000 than in 2007. However, the 2007 June Swift and XMM-Newton measurements suggest some rebrightening. The origin of the relatively large deviation in the UVW1 magnitude during the 2007 XMM-Newton observation is not obvious. The five reference stars listed in Table 2 show a large scatter between the XMM-Newton and Swift observations ranging from 0.47 to $0.96 \mathrm{mag}$. However, they all suggest that the UVW1 magnitudes between the OM and UVOT have to be adjusted significantly.

As mentioned in Grupe et al. (2007b), the optical-to-X-ray spectral slope $\alpha_{\text {ox }}{ }^{8}$ of Mrk 355 was between 1.9 and 1.65 during the $\mathrm{X}$-ray flux low state. According to the relation given in Strateva et al. (2005), $\alpha_{\text {ox }}=1.3$ is expected, which is exactly what has been seen during the high state (Grupe et al. 2007b; Gallo 2006).

\subsubsection{Optical Spectroscopy}

The top panel of Figure 10 displays the optical spectrum of Mrk 335 taken on 2007 September 7 at the Xinglong Observatory. The spectrum appears nearly identical compared to the spectrum taken 8 years earlier at the $1.52 \mathrm{~m}$ telescope at ESO La Silla

\footnotetext{
${ }^{8}$ The X-ray loudness is defined by Tananbaum et al. (1979) as $\alpha_{\text {ox }}=$ $-0.384 \log \left(f_{2 \mathrm{keV}} / f_{2500 \AA}\right)$.
}

(Grupe et al. 2004c). The continuum slope and emission-line fluxes are consistent within the errors. In Table 7 line measurements are given for features between the He II $\lambda 4686$ and $\mathrm{Fe} x$ $\lambda 6375$ emission lines. The line measurements in the $\mathrm{H} \beta$ and [O III] $\lambda 5007$ lines are, within the errors, the same as given by Grupe et al. (2004c).

Mrk 335 displays strong coronal Fe lines of [Fe VII] $\lambda 5159$, [Fe XIV] $\lambda 5303$, [ Fe VII] $\lambda \lambda 5721,6087$, and [Fe x] $\lambda 6375$ (Fig. 10). Even though coronal Fe lines are found often in Seyfert 1 galaxies (e.g., Erkens et al. 1997; Mullaney \& Ward 2008), seeing these optical lines so strong, as in Mrk 335, is rather rare. Similarly strong coronal $\mathrm{Fe}$ lines have been found in the NLS1 galaxies Mrk 1239 and Ark 564 (Erkens et al. 1997) and in the Seyfert 1.5 MCG -06-15-30 (Reynolds et al. 1997). Unusual for an NLS1, Mrk 335 also exhibits strong He II $\lambda 4686$ and He $\mathrm{I} \lambda 5876$ lines in the present spectrum, which was also detected previously (e.g., Kassebaum et al. 1997). Typically, this feature is faint in NLS1 galaxies (e.g., Grupe et al. 2004c).

\section{DISCUSSION}

We reported the XMM-Newton observations of the NLS1 Mrk 335 obtained in 2007 July 10, confirming the low state of Mrk 335 found from Swift observations in 2007 May/June (Grupe et al. 2007a). Mrk 335 is remarkable in the sense of being still bright enough in its low state to allow a detailed spectral analysis.

The X-ray spectra of a substantial fraction of (NL)S1 galaxies are complex (e.g., Gallo 2006), and different types of models have been considered to explain them, including reflection (e.g., Ballantyne et al. 2001; Crummy et al. 2006), partial covering (e.g., Tanaka et al. 2005), and relativistically smeared ionized absorption (e.g., Done et al. 2007). Variability induced by (cold) absorption is quite common in AGNs and most frequently observed in Seyfert 2 galaxies and intermediate-type Seyferts. An extreme example is the galaxy NGC 1365, which shows highly variable cold absorption along the line of sight on the timescale of days (Risaliti et al. 2007).

Partial covering models have been frequently applied to the X-ray spectra of AGNs and NLS1 galaxies in particular (e.g., Grupe et al. 2004b, 2004c; Gallo et al. 2004; Pounds et al. 2004; Tanaka et al. 2005) and also to Galactic X-ray binaries (e.g., Brandt et al. 1996; Ding et al. 2006). However, origin and confinement of the required dense blobs of gas in AGN partial coverers are not well understood (e.g., Kuncic et al. 1997). Reflection models generally provide similarly successful X-ray spectral fits. Apart from spectral modeling, temporal variability is an important discriminant between these two types of models. The deep low state of Mrk 335 discovered with Swift (Grupe et al. 2007a), and still present during our follow-up XMM-Newton TOO observation, enables us to do a detailed comparison of partial covering and reflection models.

\subsection{X-Ray Continuum}

Before we compare the models themselves, we compare Mrk 335 with several potentially similar NLS1 galaxies. The lowstate X-ray spectrum of Mrk 335 looks in many ways like the one seen during the low state in NGC 4051 (Guainazzi et al. 1998). While Pounds et al. (2004) argue for a partial covering absorber scenario, Ponti et al. (2006) argue for a reflection-dominated spectrum. While a fit to the mean low-state spectrum of NGC 4051 using a partial covering or a reflection model results statistically in very similar fits, Ponti et al. (2006) could show that a reflection model can reproduce their findings in the rms spectra and fluxflux plots better than a partial covering absorber model. Due to the relatively short length of our XMM-Newton ToO observation, 

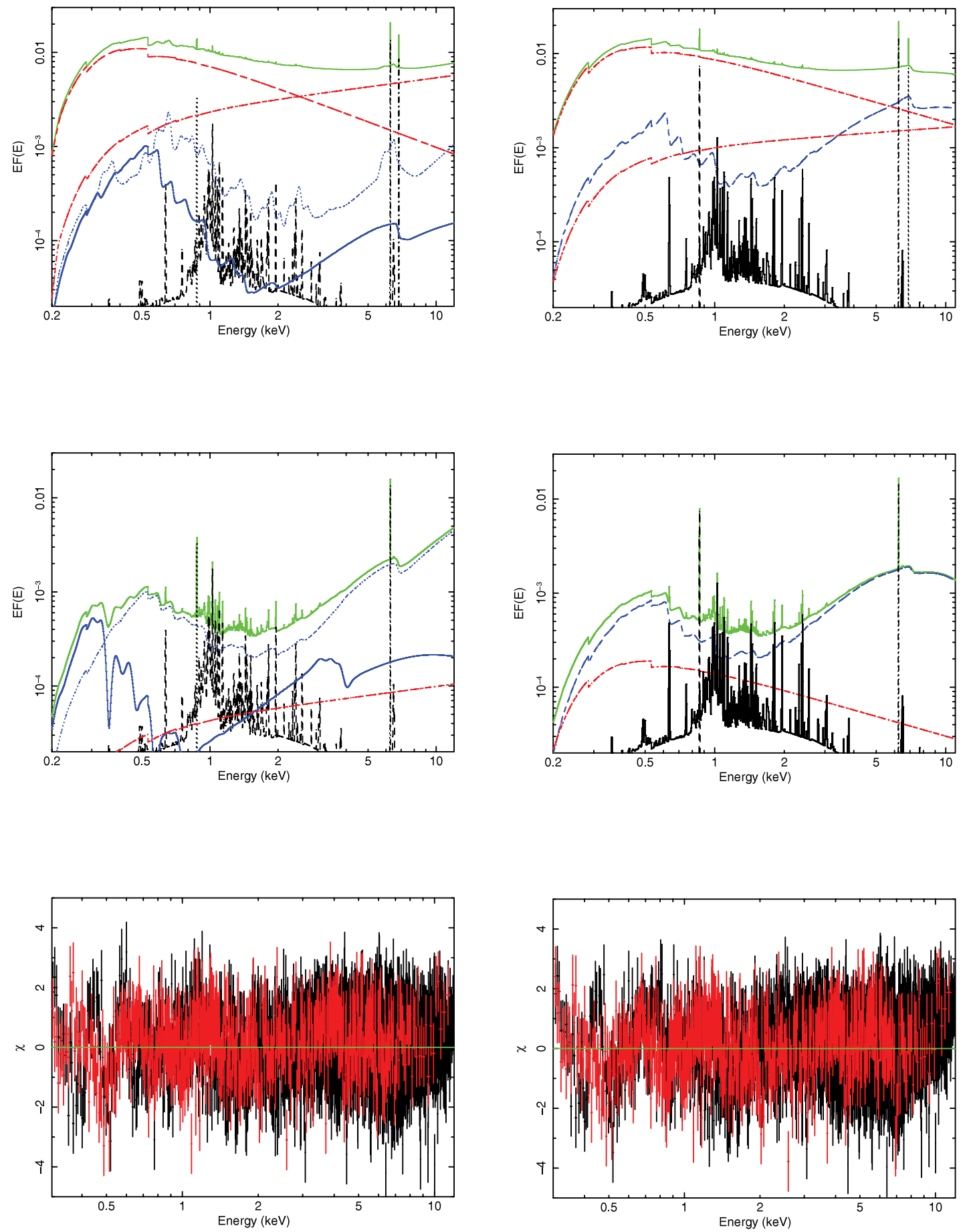

FIG. 7.-Fits to the 2006 (high state) and 2007 (low state) XMM-Newton pn spectra with reflection models. The left panels display the fits using model A (i.e., two power-law components both with associated reflection), and the right panels for model B (i.e., two power-law components, of which only one [the flatter] with associated reflection). The top panels display the fits to the high state, the middle panels the low state, and the bottom panels the residuals of the model fits. The line spectra (MEKAL+ emission lines) are in black, power laws are shown in red, both reflection components are in blue, and the total model is in green. The spectral parameters of the joint fits in the $0.3-12.0 \mathrm{keV}$ band are given in Table 6 .

the low-state data of Mrk 335 are not of sufficient quality to do such an analysis.

Mrk 335 also displays many similarities to the NLS1 galaxy Mrk 1239: (1) an unusual X-ray continuum spectrum, (2) strong X-ray emission lines (Grupe et al. 2004b), and (3) strong optical Fe coronal lines (Erkens et al. 1997). The X-ray spectrum of Mrk 1239 was interpreted by Grupe et al. (2004b) with a partial covering absorber. While Mrk 1239 is highly polarized with more than $4 \%$ at optical wavelengths (Goodrich 1989; Grupe et al. 2004b), Mrk 335 does not show any significant degree of polarization (Berriman 1989; Berriman et al. 1990; Smith et al. 2002).

The column density of the partial coverer we infer from our spectral fits is similar to those often observed in broad absorption line (BAL) quasars (e.g., Grupe et al. 2003). In this context, it is interesting to point out the potential similarity of Mrk 335 with the highly variable NLS1 galaxy WPVS 007 , which dropped by a factor of more than 400 between the RASS observation and 
TABLE 6

Summary of the Most Important Parameters a for the Best-fitting Reflection Models to the High and Low Flux State Observations

\begin{tabular}{|c|c|c|c|c|}
\hline Parameter & HF State & LF State & Tied $^{\mathrm{b}}$ & Units \\
\hline \multicolumn{5}{|c|}{ Model A $\left(\chi_{r}^{2}=1.06\right)$} \\
\hline$\alpha_{1}$ & $1.86_{-0.02}^{+0.04}$ & $1.86_{-0.02}^{+0.04}$ & $\mathrm{Y}$ & \\
\hline$F_{\alpha_{1}}$ & $(5.86 \pm 0.05) \times 10^{-11}$ & $<3.5 \times 10^{-13}$ & $\mathrm{~N}$ & {$\left[\times 10^{-3} \mathrm{~W} \mathrm{~m}^{-2}\right]=\left[\operatorname{ergs~s}^{-1} \mathrm{~cm}^{-2}\right]$} \\
\hline$\alpha_{2}$ & $0.70 \pm 0.01$ & $0.70 \pm 0.01$ & $\mathrm{Y}$ & \\
\hline 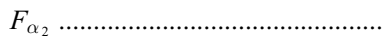 & $(1.93 \pm 0.01) \times 10^{-11}$ & $(6.9 \pm 1.3) \times 10^{-13}$ & $\mathrm{~N}$ & {$\left[\times 10^{-3} \mathrm{~W} \mathrm{~m}^{-2}\right]=\left[\operatorname{ergs~s}^{-1} \mathrm{~cm}^{-2}\right]$} \\
\hline$r_{\text {in }}$ & $1.6 \pm 0.1$ & $1.6 \pm 0.1$ & $\mathrm{Y}$ & $r_{g}^{\mathrm{c}}$ \\
\hline i & $39 \pm 1$ & $39 \pm 1$ & $\mathrm{Y}$ & $\operatorname{deg}$ \\
\hline$q_{1}$ & $3.6_{-0.6}^{+0.3}$ & $3.6_{-0.6}^{+0.3}$ & $\mathrm{Y}$ & $\ldots$ \\
\hline$q_{2}$ & $4.3 \pm 0.3$ & $4.3 \pm 0.3$ & $\mathrm{Y}$ & $\ldots$ \\
\hline$r_{\mathrm{br}}$ & $45_{-18}^{+26}$ & $>1.8$ & $\mathrm{~N}$ & $r_{g}^{\mathrm{c}}$ \\
\hline$\xi_{1}$ & $150_{-15}^{+28}$ & $38_{-1}^{+2}$ & $\mathrm{~N}$ & {$\left[\times 10^{-3} \mathrm{~W} \mathrm{~m}^{-2}\right]=\left[\operatorname{ergs~s}^{-1} \mathrm{~cm}^{-2}\right]$} \\
\hline$F_{\text {ref }_{1}} \ldots \ldots \ldots \ldots$ & $(3.3 \pm 0.9) \times 10^{-12}$ & $(1.1 \pm 0.5) \times 10^{-12}$ & $\mathrm{~N}$ & {$\left[\times 10^{-3} \mathrm{~W} \mathrm{~m}^{-2}\right]=\left[\operatorname{ergs~s}^{-1} \mathrm{~cm}^{-2}\right]$} \\
\hline$\xi_{2} \ldots \ldots \ldots \ldots \ldots$ & $150_{-15}^{+28}$ & $38_{-1}^{+2}$ & $\mathrm{~N}$ & {$\left[\times 10^{-3} \mathrm{~W} \mathrm{~m}^{-2}\right]=\left[\operatorname{ergs~s}^{-1} \mathrm{~cm}^{-2}\right]$} \\
\hline$F_{\text {ref }_{2}} \ldots \ldots$ & $(3.9 \pm 0.1) \times 10^{-12}$ & $(6.4 \pm 0.4) \times 10^{-12}$ & $\mathrm{~N}$ & {$\left[\times 10^{-3} \mathrm{~W} \mathrm{~m}^{-2}\right]=\left[\operatorname{ergs~s}^{-1} \mathrm{~cm}^{-2}\right]$} \\
\hline$A_{\mathrm{Fe}_{\mathrm{l}}=2}$ & $1.6 \pm 0.1$ & $1.6 \pm 0.1$ & $\mathrm{Y}$ & solar \\
\hline \multicolumn{5}{|c|}{ Model B $\left(\chi_{r}^{2}=1.10\right)$} \\
\hline$\alpha_{1}$ & $1.64_{-0.02}^{+0.04}$ & $1.64_{-0.02}^{+0.04}$ & $\mathrm{Y}$ & \\
\hline$F_{\alpha_{1}} \ldots \ldots \ldots \ldots$ & $(4.55 \pm 0.01) \times 10^{-11}$ & $(9.7 \pm 5.1) \times 10^{-14}$ & $\mathrm{~N}$ & {$\left[\times 10^{-3} \mathrm{~W} \mathrm{~m}^{-2}\right]=\left[\operatorname{ergs~s}^{-1} \mathrm{~cm}^{-2}\right]$} \\
\hline$\alpha_{2} \ldots \ldots \ldots \ldots$ & $0.87 \pm 0.01$ & $0.74 \pm 0.01$ & $\mathrm{~N}$ & \\
\hline$F_{\alpha_{2}} \ldots \ldots$ & $(5.87 \pm 0.09) \times 10^{-12}$ & $(6.27 \pm 0.63) \times 10^{-13}$ & $\mathrm{~N}$ & {$\left[\times 10^{-3} \mathrm{~W} \mathrm{~m}^{-2}\right]=\left[\operatorname{ergs~s}^{-1} \mathrm{~cm}^{-2}\right]$} \\
\hline$r_{\text {in }}$ & $<1.34$ & $<1.34$ & $\mathrm{Y}$ & $r_{g}^{\mathrm{c}}$ \\
\hline 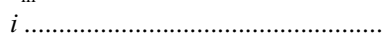 & $50 \pm 1$ & $50 \pm 1$ & $\mathrm{Y}$ & $\stackrel{9}{\operatorname{deg}}$ \\
\hline$q_{\text {in }}$ & $>4.5$ & $5.9 \pm 0.2$ & $\mathrm{~N}$ & $\ldots$ \\
\hline 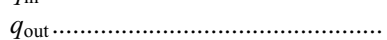 & $>4.5$ & $4.4 \pm 0.2$ & $\mathrm{~N}$ & $\ldots$ \\
\hline$r_{\mathrm{br}}$ & $2.8 \pm 0.1$ & $2.8 \pm 0.2$ & $\mathrm{~N}$ & $r_{g}^{\mathrm{c}}$ \\
\hline & $20 \pm 1$ & $24 \pm 1$ & $\mathrm{~N}$ & {$\left[\times 10^{-3} \mathrm{~W} \mathrm{~m}^{-2}\right]=\left[\operatorname{ergs~s}^{-1} \mathrm{~cm}^{-2}\right]$} \\
\hline$F_{\text {ref }}$ & $(1.23 \pm 0.01) \times 10^{-11}$ & $(5.6 \pm 0.1) \times 10^{-12}$ & $\mathrm{~N}$ & {$\left[\times 10^{-3} \mathrm{~W} \mathrm{~m}^{-2}\right]=\left[\operatorname{ergs~s}^{-1} \mathrm{~cm}^{-2}\right]$} \\
\hline$A_{\mathrm{Fe}_{1=2}} \ldots \ldots \ldots \ldots$ & $0.9 \pm 0.1$ & $0.9 \pm 0.1$ & $\mathrm{Y}$ & solar \\
\hline
\end{tabular}

${ }^{\text {a }}$ All disk radii are given in units of the gravitational radius, and the outer disk radius is fixed to $400 r_{\mid \mathrm{rmg}}$. The temperature of the MEKAL component is $1.4 \mathrm{keV}$. Other parameters listed are fluxes $F$, the inclination angle $i$, the Fe abundance $\left(A_{\mathrm{Fe}}\right)$ given relative to solar, the ionization parameter $(\xi)$, and the emissivity index $(q)$.

b This column marks which model parameters have been tied between the high- and low-state spectra.

c Gravitational radius $r_{g}=G M / c^{2}$.

follow-up X-ray observations (Grupe et al. 1995, 2007b). Neither NLS1 galaxy shows any detectable changes in their optical spectra even though their X-ray fluxes changed substantially. While Mrk 335 lacks UV spectroscopy during the low state, the UV spectrum of WPVS 007 showed the emergence of strong BAL

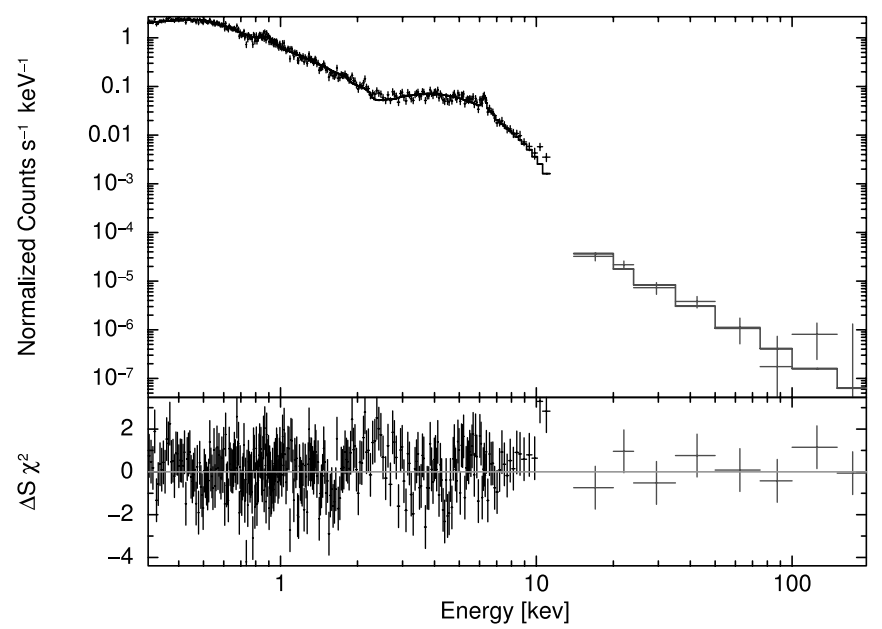

FIG. 8.-Combined XMM-Newton pn and Swift BAT spectrum fitted with a power law with partial covering absorber. [See the electronic edition of the Journal for a color version of this figure.] absorption features (K. M. Leighly et al. 2008, in preparation). Recent Swift observations of WPVS 007 suggest that the X-ray spectrum is described by a partial covering absorber (D. Grupe et al. 2008, in preparation).

An interesting connection can also be drawn to the X-ray weak quasar PG 2112+059 (Schartel et al. 2007). Also here the X-ray spectra can be fitted by partial covering absorber and reflection models. Although the fits are statistically similar (like what we saw in Mrk 335), the authors prefer the blurred X-ray ionized reflection model because the absorber model yields unreasonably high column densities and ionization parameters. In general, studying bright AGNs in low state like Mrk 335 gives us access to well-exposed X-ray spectra of X-ray-weak AGNs, which appear to be significantly fainter in X-rays than what is expected from optical luminosities (Brandt et al. 2000). Typically these AGNs are too faint in X-rays to obtain well-exposed X-ray spectra. Note that the situation in Mrk 335 is different compared with the X-ray-weak quasar PHL 1811, which is intrinsically X-ray weak (Leighly et al. 2007).

Spectral fits.-The spectral fits to the X-ray spectra of Mrk 335 involving a partial coverer are relatively simple, in the sense of only involving two free parameters to describe the partial coverer itself. All low-state spectra of Mrk 335 can be fitted by the same underlying single power law with $\alpha_{\mathrm{X}}=1.8$. However, the $X M M$ Newton high-state data cannot be fitted by the same underlying power law. The 2006 XMM-Newton data do require an underlying 

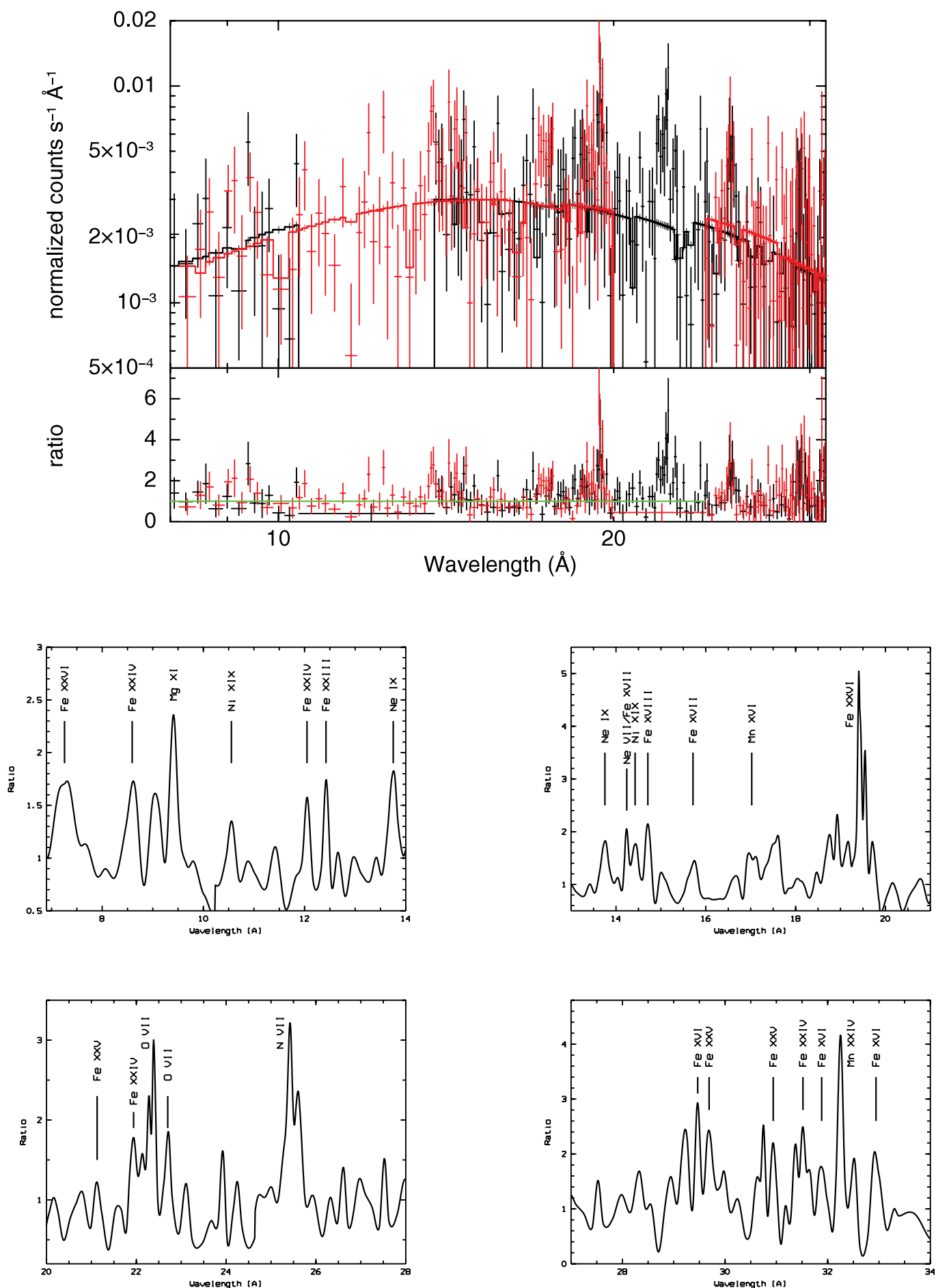

FIG. 9.- RGS spectra of Mrk 335. The top panel displays the RGS spectra fitted by a single power-law model. Clearly the residuals reveal several soft X-ray emission lines. The lower panels display the theoretical positions of some expected emission lines that are marked in the spectrum at different wavelength ranges.

broken power-law model (Grupe et al. 2007a). We performed several tests with double power law with one and two partial covering absorbers. We found that we can fit the high and low states with the same underlying continuum model, but the lowstate spectrum then requires a very high column density of several times $10^{24} \mathrm{~cm}^{-2}$ to describe the spectrum at energies $>2 \mathrm{keV}$. On the other hand, a constant flux of the underlying continuum may explain why the optical spectrum has not changed at all between 1999 and 2007.
The steep X-ray spectral slope $\alpha_{\mathrm{X}}=1.8$ continues even into the Swift BAT hard X-ray energy range. While X-ray spectral slopes of $\alpha_{X}=1.8$ are quite common for NLS1s in the $0.2-$ $2.0 \mathrm{keV}$ range (Boller et al. 1996; Grupe et al. 2001), this steep $\mathrm{X}$-ray spectral slope is unusual at hard X-ray energies. In the BAT survey (Tueller et al. 2008) no AGN has been found with an $\mathrm{X}$-ray spectral slope as steep as Mrk 335. Note that also in the $2-10 \mathrm{keV}$ range an X-ray spectral slope $\alpha_{\mathrm{X}}=1.8$ is quite unusual (Brandt et al. 1997; Leighly 1999b). As suggested by, e.g., 

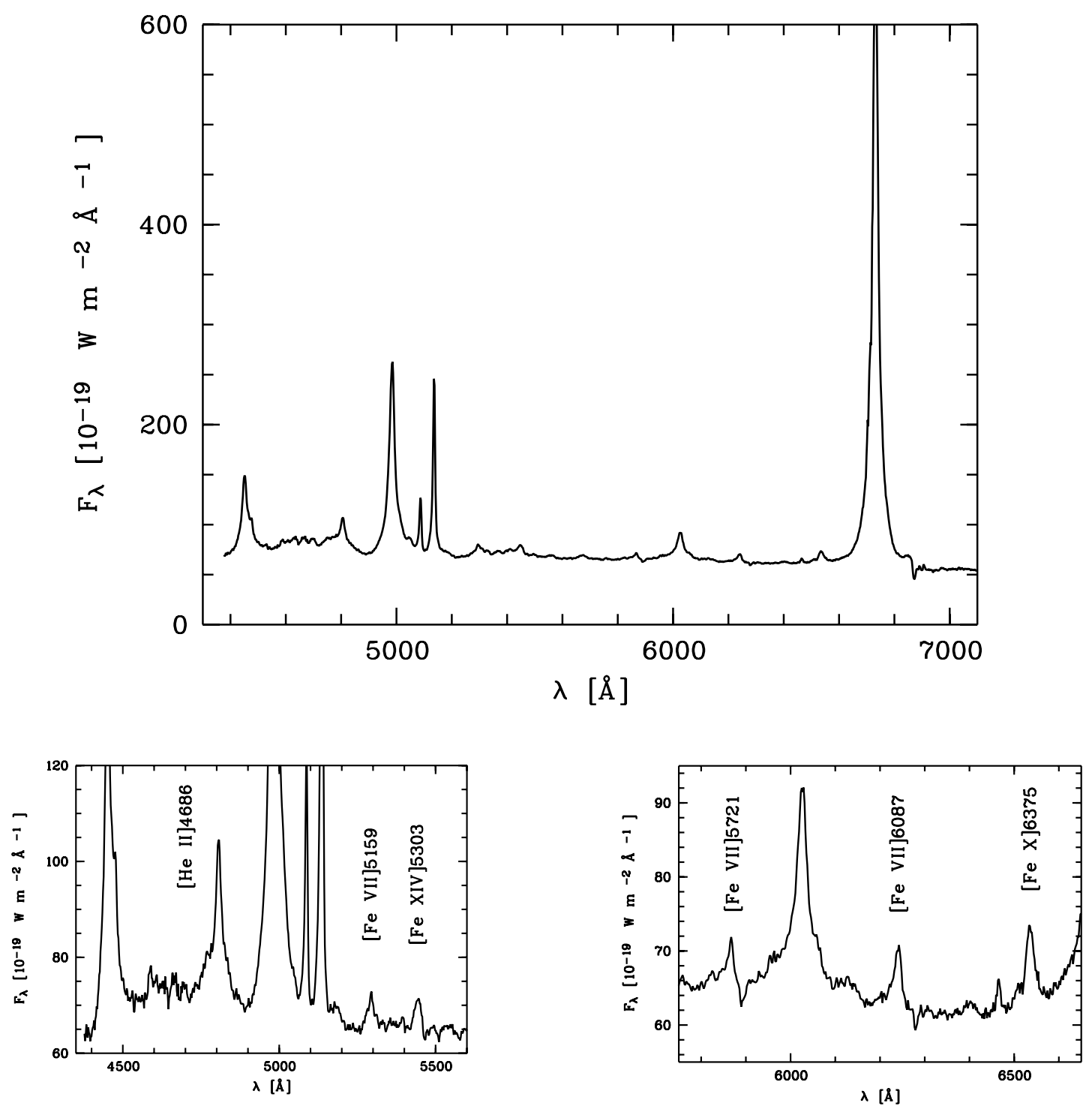

FIG. 10.- Optical spectrum of Mrk 335. The top panel displays the whole optical spectrum taken on 2007 September 7. The bottom two panels show the blue and red regions of the highly ionized coronal Fe line in the Fe II-subtracted optical spectrum of Mrk 335.

TABLE 7

Measurements of the Optical Emission Lines

\begin{tabular}{|c|c|c|c|c|}
\hline Line & $\begin{array}{l}\text { EW } \\
(\AA)\end{array}$ & $\begin{array}{c}\lambda_{c, \mathrm{obs}} \\
(\AA)\end{array}$ & $\begin{array}{c}\text { FWHM } \\
\left(\mathrm{km} \mathrm{s}^{-1}\right)\end{array}$ & $x /[\mathrm{O} \mathrm{III}]^{\mathrm{a}}$ \\
\hline He II $\lambda 4686 \mathrm{n}$ & 5.0 & 4807 & 950 & 0.19 \\
\hline He II $24686 b$ & 16.9 & 4799 & 5100 & 0.59 \\
\hline $\mathrm{H} \beta \mathrm{n}$ & 4.0 & 4984.2 & 560 & 0.13 \\
\hline $\mathrm{H} \beta \mathrm{b}$ & 90.0 & 4984.2 & 1700 & 3.23 \\
\hline [O III] $\lambda 4959 \ldots \ldots \ldots \ldots \ldots \ldots \ldots . .$. & 8.4 & 5086.7 & 500 & 0.31 \\
\hline 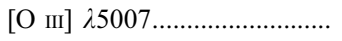 & 28.1 & 5136.1 & 500 & 1.00 \\
\hline [Fe VII] $\lambda 5159 \ldots \ldots \ldots \ldots \ldots \ldots$ & 3.4 & 5293.3 & 1250 & 0.11 \\
\hline 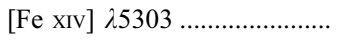 & 2.8 & 5441.3 & 1350 & 0.09 \\
\hline [Fe VII] $\lambda 5721 \ldots \ldots \ldots \ldots \ldots . . .$. & 4.0 & 5866.7 & 1250 & 0.14 \\
\hline He г $25876 \ldots \ldots$ & 15.8 & 6025.7 & 1420 & 0.54 \\
\hline 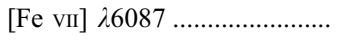 & 3.0 & 6239.6 & 930 & 0.09 \\
\hline$[\mathrm{Fe} x] \lambda 6375$ & 3.9 & 6535.8 & 900 & 0.13 \\
\hline
\end{tabular}

${ }^{\mathrm{a}}$ Line fluxes relative to $[\mathrm{O}$ III $] 25007$ line flux $\left(F_{[\mathrm{O} \text { III }}=1.93 \times 10^{-16} \mathrm{~W} \mathrm{~m}^{-2}\right)$.
Pounds et al. (1995), steep hard X-ray spectral slopes can be caused by thermal Comptonization of UV photons of the hot accretion disk corona.

$X$-ray and UV variability.-The short-time variability found during the low-state observation shows that the bulk of the soft $\mathrm{X}$-ray emission is not from an extended scattering region as suggested by Grupe et al. (2004b) for Mrk 1239 or Immler et al. (2003) for Mrk 6. These regions are typically too large to show variability on timescales of hours. Note that the mass of the central black hole in Mrk 335 is of the order of $(1.5-3) \times 10^{7} M_{\odot}$ (Peterson et al. 2004; Vestergaard \& Peterson 2006), which is on the high-mass end of the black hole mass distribution of NLS1 galaxies (Grupe \& Mathur 2004; Watson et al. 2007).

The variability detected during the low-state observation implies a source size of a few light-hours (see Fig. 2), which in turn requires that the partial coverer has to consist of very compact, dense clumps of gas.

Perhaps in favor of the partial covering interpretation is the variability in the UV/optical band (even though small). In the two bluest filters, W2 and M2, Mrk 335 varies stronger by $0.2 \mathrm{mag}$ than in the optical $V$ and $B$ filters. Since the UV magnitudes seem 
to follow the X-ray count rates, this might indicate that the variability in the UV and in X-rays is caused by the same mechanism. However, longer term monitoring of the UV and X-ray variability is required to put this statement on a more firm basis.

Models of partial coverers.-In summary, the most successful phenomenological partial coverer model consists of very compact blobs of gas covering the variable X-ray source and is mostly located along our line of sight in order to ensure nonvariable broad-line region (BLR) line emission and in order to be consistent with the absence of a detectable strongly variable $6.4 \mathrm{keV}$ iron line from the partial coverer itself. The requirement of the compactness of the clouds, in combination with their high column density, emphasizes well-known challenges to the partial coverer scenario, including questions like, what is the origin of the blobs, and how are they confined (e.g., Guilbert \& Rees 1988; Celotti et al. 1992; Kuncic et al. 1997)? In the model of Abrassart \& Czerny (2000), fragmentation of the inner accretion disk leads to the formation of compact clouds that can partially obscure the central X-ray source, confined perhaps by strong magnetic fields (Kuncic et al. 1997). These clouds would reprocess some radiation into the optical and UV band (Celotti et al. 1992; Kuncic et al. 1997; Abrassart \& Czerny 2000). If their emission does contribute to the total UV emission, then the variability in the X-ray band would be higher than that in the UV band, as observed.

Reflection models.-Blurred reflection models invoking a single continuum source and reflecting medium have been shown to fit the 2000 high flux state observation (Crummy et al. 2006) and the 2007 low flux state observation $(\S 3.2 .2)$. However, there are a number of discrepancies between parameter values (e.g., inclination and iron abundance) that indicate that these models are not consistent. Likewise, the high signal-to-noise ratio 2007 spectrum of Mrk 335 cannot be modeled self-consistently in the high- and low-energy ranges (O'Neill et al. 2007; § 3.2.2).

The fact that the low-flux spectra of Mrk 335 cannot be fitted with the same reflection model used for the higher flux states suggests that the most straightforward application of light bending does not necessarily apply (Miniutti et al. 2003; Miniutti \& Fabian 2004). That is, the low flux state is not solely due to a single continuum source whose intensity appears diminished because of its proximity to the black hole while the reflection is kept constant. One possibility is that the entire disk does not "see" the same continuum source. Such a scenario was proposed by Gallo et al. (2007a, 2007b) to describe the behavior of another NLS1, I Zw 1. Here we considered this possibility for Mrk 335.

Two models are suggested, both rendering equally good fits. Both scenarios incorporate two power-law components, each one illuminating different parts of the accretion disk. One component could be associated with a jet producing either beamed (model B) emission with no reflection component or unbeamed (model A; see Ghisellini et al. 2004) emission that preferentially illuminates the innermost region of the disk. A jet seen in Mrk 335 is plausible. Mrk 335 has a clear detection of a compact source at $4.8 \mathrm{GHz}$ (Leipski et al. 2006). The second power law is associated with the traditional idea of the corona, which blankets the disk to larger radii. These, of course, are not exclusive interpretations. The two different continuum slopes may in fact be due to different mechanisms (e.g., bulk motion Comptonization and standard thermal Comptonization; see, e.g., Niedzwiecki \& Zdziarski 2006).

Statistically, both models fit the data very well $\left(\chi_{\nu}^{2}=1.06-\right.$ $1.10)$, but more importantly in a self-consistent manner. The primary difference between the high and low flux states is the diminished flux received by the observer from the inner powerlaw component (i.e., the one perhaps associated with a jet).
According to our models A and B, there are situations (especially in the low state) when the X-ray spectrum of Mrk 335 is reflection dominated. If so, the observed flux cannot be used in a simple way to infer the intrinsic X-ray luminosity of the AGN. In general, the power-law component contributes to the observed flux at infinity, and it also illuminates the accretion disk giving rise to the associated reflection component. The intrinsic luminosity of that power law must take into account not only the flux observed at infinity but also that needed to produce the reflection component. If $L_{\mathrm{obs}}$ is the power-law luminosity observed at infinity and $R$ is the reflection fraction of the associated reflection component, the intrinsic power-law luminosity is $L_{\mathrm{int}}=$ $L_{\text {obs }}(R+1) / 2$, which, in the isotropic case $(R=1)$, simply reduces to $L_{\text {obs }}$. Applying the above arguments to model A, we estimate that the intrinsic luminosity of all power-law components is $\sim 2.3 \times 10^{43} \mathrm{ergs} \mathrm{s}^{-1} \mathrm{~cm}^{-2}$ in the high flux state and $\geq 1.9 \times 10^{43} \mathrm{ergs} \mathrm{s}^{-1} \mathrm{~cm}^{-2}$ in the low flux state (all luminosities are unabsorbed and given in the $2-10 \mathrm{keV}$ band). In other words, the intrinsic luminosity of the AGN would be similar in the two states despite the dramatic observed flux variation.

In the case of model B, we have one power law with its reflection (for which the same arguments as above can be applied) and one power law with no associated reflection. The latter could be due to a jetted component beaming the radiation away from the disk (so that there is no reflection). Hence, we can estimate its intrinsic luminosity by assuming that the radiation is beamed in the half-sphere away from the disk $\left(L_{\mathrm{int}}<=L_{\mathrm{obs}} / 2\right)$. For model B, considering the contributions of both the jetted and the nonjetted components, we then obtain intrinsic luminosities in the range $(6-6.8) \times 10^{43} \mathrm{ergs} \mathrm{s}^{-1} \mathrm{~cm}^{-2}$ in the high flux state and $\sim 2.4 \times$ $10^{43} \mathrm{ergs} \mathrm{s}^{-1} \mathrm{~cm}^{-2}$ in the low flux state (the jetted contribution is negligible in the low flux state).

Partial covering absorber versus reflection models.-Statistically the reflection models yield better fits compared with the partial covering absorber model (reduced $\chi^{2}=1.10$ vs. 1.27, respectively). Both the partial covering absorber model and the reflection models require underlying broken power-law or double power-law model spectra to fit the high states of Mrk 335. For both models the same underlying continua can be used in the high and low states, just modified by either absorption or reflection. The parameters, however, used for the partial covering absorber model then require that the absorption column density of one of the partial covering absorber components is large on the order of several times $10^{24} \mathrm{~cm}^{-2}$. Even though this is not impossible, it requires extremely dense compact clouds to pass through the line of sight.

Reflection models have been used in the past to describe the X-ray spectra of many AGNs (e.g., Fabian 2008) and have also been applied successfully to describe the high-state X-ray spectra of Mrk 335 (e.g., Ballantyne et al. 2001; Crummy et al. 2006; Longinotti et al. 2007b; O'Neill et al. 2007), and we have shown here that they also consistently produce the low state. Further, we mention in passing that we cannot exclude a third possibility: that the spectrum is dominated in high state by reflection, and this reflection spectrum is modified by partial covering absorption. A scenario like this has been suggested, e.g., by Chevallier et al. (2006) and Merloni et al. (2006). In some ways the situation in Mrk 335 is similar to that of PG 2112+059, where the absorber model also yields very high column densities, which seems to favor the reflection model. On the other hand, the partial covering absorber model is rather simple and does not require many parameters to fit the data reasonably well. At the end one has to conclude that both models are valid to explain the spectral shape seen in the low-state spectrum of Mrk 335. Nevertheless, with 
the low-state XMM-Newton observation presented here we were able to put strong constraints on both the partial covering and reflection model parameters. In order to really distinguish between both models, either a very long XMM-Newton observation in low state is needed to search for the deviation between both models at intermediate energies between 2 and $6 \mathrm{keV}$ or it is necessary to obtain a spectrum at energies $>10 \mathrm{keV}$ using, e.g., Suzaku.

\subsection{X-Ray Emission Lines}

Several low-energy $(E<2 \mathrm{keV}) \mathrm{X}$-ray emission lines are detected in the RGS spectra of Mrk 335. Soft X-ray emission lines are typically found in obscured Seyfert 2 type AGNs (e.g., Guainazzi \& Bianchi 2007; Kinkhabwala et al. 2002) and are only seen in Seyfert 1 galaxies when the AGN enters a low flux state. Consequently, low-energy emission lines have only been detected in a few Seyfert 1 galaxies like Mrk 590, NGC 4051, and NGC 5548 (Longinotti et al. 2007a; Pounds et al. 2004; Steenbrugge et al. 2003, respectively). This is the first time such lines have been observed in Mrk 335. Even though these lines are expected in Seyfert 1 galaxies when the continuum is suppressed, as in the low-state 2007 XMM-Newton observation, they are very prominent even with just a $22 \mathrm{ks}$ RGS exposure.

Most notable in the RGS spectrum of Mrk 335 is the $\mathrm{O}$ VII emission line complex around $22 \AA$. The forbidden (f) line at $22.1012 \AA$ and the intercombination (i) line at $21.8044 \AA$ are secure identifications. These lines have also been found in the low-state RGS spectra of NGC 4051 (Pounds et al. 2004; Ponti et al. 2006). The resonance (r) line appears to be weak and slightly redshifted with respect to its rest-frame wavelength of $21.602 \AA$, which could be due to resonance scattering and absorption. Although the line intensities are considerably uncertain, the presence of the $\mathrm{O}$ VII line complex suggests coronal emission from collisionally ionized plasma. In principle, the lines from this complex can yield reasonably precise information on the temperature, densities, and ionization state of the source. However, owing to the uncertainties, we conclude that it is not useful at this time to attempt a detailed line ratio analysis such as the $G=$ $(i+f) / r$ ratio or the $R=f / i$ ratio (e.g., Pradhan \& Shull 1981). Nor is it possible to deduce the existence of additional features widely seen in warm absorber spectra (e.g., Lee et al. 2001), such as the $\mathrm{O}$ vi KLL absorption features at $(22.05,21.87) \AA$, lying between the $f$ and $i$ lines (Pradhan 2000; Pradhan et al. 2003). In addition to $\mathrm{O}$ vII, we have identified, to varying degree of certainty, a number of iron lines from coronal ions such as Fe xvII to Fe xxv. The iron lines complement the independent observations of the UV/optical lines from coronal iron ions in lower ionization stages discussed in the next section. Note that the RGS data have independently been analyzed by Longinotti et al. (2008), who present line ratios. They did not find a unique solution of the plasma properties either, but concluded that the location of the $\mathrm{X}$-ray line-emitting gas is within $0.01 \mathrm{pc}$.

\subsection{Optical Emission Lines}

The optical emission line spectrum of Mrk 335 is remarkably constant between the observations taken 8 years ago and the one taken nearly simultaneously with the X-ray low-state observations. Since the broad component of the Balmer lines and the broad component of the He II line are very sensitive to the EUVto-soft X-ray part of the continuum, the observed lack of variability implies that the bulk of the BLR still sees the high-state continuum. In the context of the partial covering model, this can be understood if the absorber has a small global covering fraction and is mostly located along our line of sight.

The highly ionized coronal Fe lines found in the optical spectrum of Mrk 335 are rather strong. While coronal Fe lines are present in the optical spectra of Seyfert 1 galaxies, they typically appear faint (e.g., vanden Berk et al. 2001) with typical flux ratios to $[\mathrm{O}$ III] $\lambda 5007$ of about $0.03-0.06$. In comparison with the AGN sample of Erkens et al. (1997), only 2 out of 15 AGNs (Mrk 486 and Ark 564) have coronal Fe lines stronger than Mrk 335. The central wavelengths of the iron lines in Mrk 335 suggest that they are blueshifted with respect to the [O III] lines, as is commonly seen in coronal lines (e.g., Erkens et al. 1997; Rodriguez-Ardila et al. 2006). The $5 \AA$ resolution of our spectrum, however, does not allow a precise measurement of the central wavelength of the lines; therefore, the shift of the line with respect to the rest frame cannot be determined with high accuracy. As generally observed (Erkens et al. 1997), the line widths of the coronal $\mathrm{Fe}$ lines are between the narrow-line region $(\mathrm{FWHM} \approx$ $\left.500 \mathrm{~km} \mathrm{~s}^{-1}\right)$ and the BLR $\left[\mathrm{FWHM}(\mathrm{H} \beta)=1700 \mathrm{~km} \mathrm{~s}^{-1}\right]$.

\section{CONCLUSIONS}

We report the XMM-Newton and Swift observations of the bright NLS1 galaxy Mrk 335 in a deep, and unprecedented, low flux state. The main findings are as follows:

1. The XMM-Newton ToO observation of 2007 July 10 still found Mrk 335 in a very low flux state as previously detected by Swift. With this XMM-Newton observation, however, the parameters of the X-ray spectral models can be well constrained. Low-state observations of Seyfert 1 galaxies are rare, and Mrk 335 is exceptional in being still bright in its low state. Low states give us access to X-ray emission lines that otherwise cannot be seen (see below). The low state of Mrk 335 lasted at least 2 months.

2. While a simple broken power-law model requires a broad redshifted $\mathrm{Fe}$ Gaussian line component with an extremely large equivalent width $\mathrm{EW}=2.4 \mathrm{keV}$, this line component is not required in either the partial covering absorber or the reflection models. The partial covering model can be fitted to high-state and low-state data with the same underlying double power-law model continuum when two absorber components are used, even though formally a second power-law component is not required to fit the low-state spectrum. The reflection model formally accounts for the two-component nature of the observed spectrum by multiple reflection where different parts of the accretion disk see different continua.

3. High-resolution spectroscopy clearly reveals the presence of X-ray emission lines in the low-energy X-ray spectrum of Mrk 335, including transitions of oxygen and iron. These lines are only visible in the low state. Preliminary results suggest that the emission-line gas is collisionally ionized. However, to perform proper line diagnostics of the ionized gas to derive gas density and temperature requires a longer RGS exposure of Mrk 335 in its low state.

4. The optical spectrum of Mrk 335 taken during the X-ray low state did not change in comparison to a spectrum taken 8 years earlier. The constancy of the broad Balmer lines indicates that the bulk of the BLR was still illuminated by the "high-state" continuum. The optical spectrum of Mrk 335 displays a variety of strong, highly ionized coronal Fe lines. These might be linked to the component emitting the X-ray lines detected with RGS. The nonvariability seen in these lines may indicate that the intrinsic SED has not changed. 
Mrk 335 will continue to be monitored with Swift in order to trigger another, much deeper X-ray observation if the AGN enters another low state.

This work is based on observations obtained with XMM-Newton, an ESA science mission with instruments and contributions directly funded by ESA Member States and NASA. In Germany, the XMM-Newton project is supported by the Bundesministerium für Wirtschaft und Technologie/Deutsches Zentrum fur Luft und Raumfahrt and the Max-Planck Society. We thank the XMMNewton Project Scientist Norbert Schartel for approving our ToO request and Michel Breitfellner for preparing and scheduling the XMM-Newton observation. We are very grateful to Ron Remillard for preparing the RXTE ASM light curve, the Swift PI Neil Gehrels for approving the monitoring campaign of
Mrk 335 with Swift, and Jack Tueller for providing the 22 month BAT survey spectrum of Mrk 335. We thank the Xinglong Observatory, NAOC for providing telescope time, and the support astronomer Jing Wang and his night assistant Jianjun Jia for performing the optical spectroscopy observations on 2007 September 7. We also want to thank the anonymous referee for his/her helpful and constructive report that improved this paper. This research has made use of the NASA/IPAC Extragalactic Database (NED), which is operated by the Jet Propulsion Laboratory, Caltech, under contract with the National Aeronautics and Space Administration, and the Atomic Spectra Database (ver. 3.1.2) of the National Institute of Standards and Technology (http://physics.nist.gov/asd3). D. X. acknowledges the support of the Chinese National Science Foundation (NSFC) under grant NSFC-10503005. Swift is supported at PSU by NASA contract NAS5-00136. This research was supported by NASA contracts NNX07AH67G and NNX07AV04G (D. G.).
Abrassart, A., \& Czerny, B. 2000, A\&A, 356, 475

Arnaud, K. A. 1996, in ASP Conf. Ser. 101, Astronomical Data Analysis Software and Systems V, ed. G. H. Jacoby \& J. Barnes (San Francisco: ASP), 17

Ballantyne, D. R., Iwasawa, K., \& Fabian, A. C. 2001, MNRAS, 323, 506

Barthelmy, S. D., et al. 2005, Space Sci. Rev., 120, 143

Berriman, G. 1989, ApJ, 345, 713

Berriman, G., Schmidt, G. D., West, S. C., \& Stockman, H. S. 1990, ApJS, 74, 869

Boller, T., Brandt, W. N., \& Fink, H. H. 1996, A\&A, 305, 53

Boroson, T. A., \& Green, R. F. 1992, ApJS, 80, 109

Brandt, W. N., Fabian, A. C., Dotani, T., Nagase, F., Inoue, H., Kotani, T., \& Segawa, Y. 1996, MNRAS, 283, 1071

Brandt, W. N., Laor, A., \& Wills, B. J. 2000, ApJ, 528, 637

Brandt, W. N., Mathur, S., \& Elvis, M. 1997, MNRAS, 285, L25

Burrows, D., et al. 2005, Space Sci. Rev., 120, 165

Celotti, A., Fabian, A. C., \& Rees, M. J. 1992, MNRAS, 255, 419

Chevallier, L., Collin, S., Dumont, A.-M., Czerny, B., Mouchet, M., Gonçalves,

A. C., \& Goosmann, R. 2006, A\&A, 449, 493

Crummy, J., Fabian, A. C., Gallo, L. C., \& Ross, R. R. 2006, MNRAS, 365, 1067

den Herder, J. W., et al. 2001, A\&A, 365, L7

Dickey, J. M., \& Lockman, F. J. 1990, ARA\&A, 28, 215

Ding, G. Q., Qu, J. L., \& Li, T. P. 2006, AJ, 131, 1693

Done, C., Sobolewska, M. A., Gierlinski, M., \& Schurch, N. J. 2007, MNRAS, 374, L15

Dunn, J. P., Jackson, B., Deo, R. P., Farrington, C., Das, V., \& Crenshaw, D. M. 2006, PASP, 118, 572

Erkens, U., Appenzeller, I., \& Wagner, S. 1997, A\&A, 323, 707

Fabian, A. C. 2008, Astron. Nachr., 329, 155

Fabian, A. C., Miniutti, G., Gallo, L. C., Boller, T., Tanaka, Y., Vaughan, S., \& Ross, R. R. 2004, MNRAS, 353, 1071

Fabian, A. C., Rees, M. J., Stella, L., \& White, N. E. 1989, MNRAS, 238, 729

Gallo, L. C. 2006, MNRAS, 368, 479

Gallo, L. C., Brandt, W. N., Constantini, E., \& Fabian, A. C. 2007a, MNRAS, 377,1375

Gallo, L. C., Brandt, W. N., Constantini, E., Fabian, A. C., Iwasawa, K., \& Papadakis, I. E. 2007b, MNRAS, 377, 391

Gallo, L. C., Tanaka, Y., Boller, Th., Fabian, A. C., Vaughan, S., \& Brandt, W. N. 2004, MNRAS, 353, 1064

Gehrels, N., et al. 2004, ApJ, 611, 1005

George, I. M., Turner, T. J., Yaqoob, T., Netzer, H., Laor, A., Mushotzky, R. F., Nandra, K., \& Takahashi, T. 2000, ApJ, 531, 52

Ghisellini, G., Haardt, F., \& Matt, G. 2004, A\&A, 413, 535

Gondoin, P., Orr, A., Lumb, D., \& Santos-Lleo, M. 2002, A\&A, 388, 74

Goodrich, R. W. 1989, ApJ, 342, 224

Grupe, D., Beuermann, K., Mannheim, K., Thomas, H.-C., Fink, H. H., \& de Martino, D. 1995, A\&A, 300, L21

Grupe, D., Komossa, S., \& Gallo, L. C. 2007a, ApJ, 668, L111

Grupe, D., Leighly, K. M., Burwitz, V., Predehl, P., \& Mathur, S. 2004a, AJ, 128,1524

Grupe, D., \& Mathur, S. 2004, ApJ, 606, L41

Grupe, D., Mathur, S., \& Elvis, M. 2003, AJ, 126, 1159

Grupe, D., Mathur, S., \& Komossa, S. 2004b, AJ, 127, 3161

Grupe, D., Schady, P., Leighly, K. M., Komossa, S., O’Brien, P. T., \& Nousek, J. A. $2007 b$, AJ, 133, 1988

\section{EFERENCES}

Grupe, D., Thomas, H.-C., \& Beuermann, K. 2001, A\&A, 367, 470

Grupe, D., Wills, B. J., Leighly, K. M., \& Meusinger, H. 2004c, AJ, 127, 156 Guainazzi, M., \& Bianchi, S. 2007, MNRAS, 374, 1290

Guainazzi, M., Bianchi, S., \& Dovčiak, M. 2006, Astron. Nachr., 327, 1032

Guainazzi, M., et al. 1998, MNRAS, 301, L1

Guilbert, P. W., \& Rees, M. J. 1988, MNRAS, 233, 475

Halpern, J. P. 1982, Ph.D. thesis, Harvard Univ.

Immler, S., Brandt, W. N., Vignali, C., Bauer, F. E., Crenshaw, D. M., Feldmeier, J. J., \& Kraemer, S. B. 2003, AJ, 126, 153

Jansen, F., et al. 2001, A\&A, 365, L1

Kassebaum, T. M., et al. 1997, ApJ, 475, 106

Kinkhabwala, A., et al. 2002, ApJ, 575, 732

Kuncic, Z., Celotti, A., \& Rees, M. J. 1997, MNRAS, 284, 717

Larsson, J., Miniutti, G., Fabian, A. C., Miller, J. M., Reynolds, C. S., \& Ponti, G. 2008, MNRAS, 384, 1316

Lee, J. C., Ogle, P. M., Canizales, C. R., Marshall, H. L., Schulz, N. S., Morales, R., Fabian, A. C., \& Iwasawa, K. 2001, ApJ, 554, L13

Leighly, K. M. 1999a, ApJS, 125, 297

1999b, ApJS, 125, 317

Leighly, K. M., Halpern, J. P., Jenkins, E. B., Grupe, D., Choi, J., \& Prescott, K. B. 2007, ApJ, 663, 103

Leipski, C., Falcke, H., Bennert, N., \& Hüttemeister, S. 2006, A\&A, 455, 161 Longinotti, A. L., Bianchi, S., Santos-Lleo, M., Rodriguez-Pascual, P., Guainazzi, M., Cardaci, M., \& Pollock, A. M. T. 2007a, A\&A, 470, 73

Longinotti, A. L., Nucita, A., Santos-Lleo, M., \& Guainazzi, M. 2008, A\&A, 484,311

Longinotti, A. L., Sim, S. A., Nandra, K., \& Cappi, M. 2007b, MNRAS, 374, 237

Longinotti, A. L., Sim, S. A., Nandra, K., Cappi, M., \& O’Neill, P. 2007c, in ASP Conf. Ser. 373, The Central Engine of Active Galactic Nuclei, ed. L. C. Ho \& J.-M. Wang (San Francisco: ASP), 341

Mason, K. O., et al. 2001, A\&A, 365, L36

Merloni, A., Malzac, J., Fabian, A. C., \& Ross, R. R. 2006, MNRAS, 370, 1699

Miniutti, G., \& Fabian, A. C. 2004, MNRAS, 349, 1435

Miniutti, G., Fabian, A. C., Goyder, R., \& Lasenby, A. N. 2003, MNRAS, 344, L22

Mullaney, J. R., \& Ward, M. J. 2008, MNRAS, 385, 53

Nandra, K., O’Neill, P. M., George, I. M., \& Reeves, J. N. 2007, MNRAS, 382, 194

Nandra, K., \& Pounds, K. A. 1994, MNRAS, 268, 405

Niedzwiecki, A., \& Zdziarski, A. 2006, MNRAS, 365, 606

O’Neill, P. M., Nandra, K., Cappi, M., Longinotti, A. L., \& Sim, S. A. 2007, MNRAS, 381, L94

Osterbrock, D. E., \& Pogge, R. W. 1985, ApJ, 297, 166

Peterson, B. M., et al. 2004, ApJ, 613, 682

Ponti, G., Miniutti, G., Cappi, M., Maraschi, L., Fabian, A. C., \& Iwasawa, K. 2006, MNRAS, 368, 903

Poole, T. S., et al. 2008, MNRAS, 383, 627

Pounds, K. A., Done, C., \& Osborne, J. 1995, MNRAS, 277, L5

Pounds, K. A., Reeves, J. N., King, A. R., \& Page, K. L. 2004, MNRAS, 350, 10

Pounds, K. A., Stanger, V. J., Turner, T. J., King, A. R., \& Czerny, B. 1987, MNRAS, 224, 443 
Pradhan, A. K. 2000, ApJ, 545, L165

Pradhan, A. K., Chen, G. X., Delahaye, F., Nahar, S. N., \& Oelgoetz, 2003, MNRAS, 341, 1268

Pradhan, A. K., \& Shull, J. M. 1981, ApJ, 249, 821

Reynolds, C. S., Ward, M. J., Fabian, A. C., \& Celotti, A. 1997, MNRAS, 291, 403

Risaliti, G., Elvis, M., Fabbiano, G., Baldi, A., Zezas, A., \& Salvati, M. 2007, ApJ, 659, L111

Rodriguez-Ardila, A., Prieto, A., Viegas, S., \& Gruenwald, R. 2006, ApJ, 653, 1098

Roming, P. W. A., et al. 2005, Space Sci. Rev., 120, 95

Schartel, N., Rodriguez-Pascual, P. M., Santos-Lleo, M., Ballo, L., Clavel, J., Guainazzi, M., Jimenez-Bailon, \& Piconcelli, E. 2007, A\&A, 474, 431

Schlegel, D. J., Finkbeiner, D. P., \& Davis, M. 1998, ApJ, 500, 525

Smith, J. E., Young, S., Robinson, A., Corbett, E. A., Giannuzzi, M. E., Axon, D. J., \& Hough, J. H. 2002, MNRAS, 335, 773

Steenbrugge, K. C., Kaastra, J. S., deVries, C. P., \& Edelson, R. 2003, A\&A, 402, 477
Strateva, I. V., Brandt, W. N., Schneider, D. P., Vanden Berk, D. E., \& Vignali, C. 2005, AJ, 130,387

Strüder, L., et al. 2001, A\&A, 365, L18

Tanaka, Y., Boller, T., \& Gallo, L. C. 2005, in Growing Black Holes: Accretion in a Cosmological Context, ed. A. Merloni et al. (Berlin: Springer), 290

Tananbaum, H., Peters, G., Forman, W., Giacconi, R., Jones, C., \& Avni, Y. 1978, ApJ, 223, 74

Tananbaum, H., et al. 1979, ApJ, 234, L9

Tueller, J., Mushotzky, R. F., Barthelmy, S., Cannizzo, J. K., Gehrels, N., Markwardt, C. B., Skinner, G. K., \& Winter, L. M. 2008, ApJ, 681, 113

Turner, M. J., et al. 2001, A\&A, 365, L27

Turner, T. J., et al. 1993, ApJ, 407, 556

Uttley, P., \& McHardy, I. M. 2001, MNRAS, 323, L26

vanden Berk, D. E., et al. 2001, AJ, 122, 549

Vestergaard, M., \& Peterson, B. M. 2006, ApJ, 641, 689

Voges, W., et al. 1999, A\&A, 349, 389

Watson, L. C., Mathur, S., \& Grupe, D. 2007, AJ, 133, 2435 